\title{
Equation of state for $\beta$-stable hot nuclear matter
}

\author{
Ch.C. Moustakidis and C.P. Panos \\ Department of Theoretical Physics, Aristotle University of Thessaloniki, \\ 54124 Thessaloniki, Greece
}

October 27, 2018

\begin{abstract}
We provide an equation of state for hot nuclear matter in $\beta$-equilibrium by applying a momentum-dependent effective interaction. We focus on the study of the equation of state of high-density and high-temperature nuclear matter, containing leptons (electrons and muons) under the chemical equilibrium condition in which neutrinos have left the system. The conditions of charge neutrality and equilibrium under $\beta$-decay process lead first to the evaluation of proton and lepton fractions and afterwards of internal energy, free energy, pressure and in total to the equation of state of hot nuclear matter. Thermal effects on the properties and equation of state of nuclear matter are assesed and analyzed in the framework of the proposed effective interaction model. Special attention is dedicated to the study of the contribution of the components of $\beta$-stable nuclear matter to the entropy per particle, a quantity of great interest for the study of structure and collapse of supernova.
\end{abstract}

PACS number(s): 21.65.+f, 21.30.Fe, 24.10.Pa, 26.60.+c, 26.50.+x

Keywards: Hot Nuclear Matter, Effective Interaction, Equation of State, Nuclear Symmetry Energy, Proton Fraction, Neutron Star.

\section{Introduction}

The equation of state (EOS) of hot nuclear matter determines the structure inside a supernova [1] and a hot neutron star [2, 3, 4] and affects the state of matter, such as its chemical composition. In addition, the equation of state plays important roles for the study of the supernova explosion, as well as on determining the evolution of a neutron star at the birth stage. The profiles of a neutron star as the density, temperature and proton fraction during the cooling, which affect the reaction rate of neutrino process inside the star, are determined through the equation of state.

There exist many calculations for hot nuclear matter with applications to the properties of hot neutron stars and supernova [1, 2, 3, 4, 15, 6, 17, 8, 9, 10, 11, 12, 13, 14, 15, 16, 17, 18, 19, 20, 21, 22, 23, 24, 25, 26, 27, 28, 29, 30, 31, 32, 33, 34, 35, 36, 37, 38, 39, 40. G. Baym et al. provided an EOS of neutron matter [5] and Bethe et al. [6] an EOS for the gravitational collapse of stars. Friedman and Pandharipande [7] performed variational calculations of the equation of state of hot and cold, nuclear and neutron matter. Lattimer and Swesty carried out calculations of the EOS for stellar collapse, using the compressible liquid-drop model for nuclei [8]. M. Prakash et al. 2] investigated the structure of neutron stars shortly after their birth, by applying various nuclear models. Takatsuka et al. [9, 10] have performed detailed calculations for supernova matter, within the framework of finite temperature Hartree-Fock approach, with effective nucleon-nucleon 
interaction. Recently Das et al. [11] have calculated the EOS of dense supernova matter within the finite temperature Brueckner Goldstone approach with effective two-body Sussex interaction.

The present work is based on the previous work of Prakash et al. [2]. More specifically, in order to study the properties and the EOS of hot nuclear matter, we apply a momentum-dependent effective interaction model (MDIM), which is able to reproduce the results of more microscopic calculations of dense matter at zero temperature and which can be extended to finite temperature [2, 39, 40].

The aim of this work is to apply a momentum-dependent interaction model for the study of hot nuclear matter EOS under $\beta$-equilibrium. The present model has the additional property, compared to the previous ones, that the temperature affects not only the kinetic part of the energy density, but also influences the interaction part of the energy density as well. In that way, we are able to study simultaneously thermal effects not only on the kinetic part of the symmetry energy and symmetry free energy, but also on the interaction part of the above quantities. This is important in the sense that the density dependent behavior of the symmetry energy and symmetry free energy influence strongly the values of the proton fraction and as a consequence the composition of hot $\beta$-stable nuclear matter, under consideration.

Using the above method, we will show that the thermal energy (and also the related quantities) depend sensitively on the momentum dependence of the nuclear interaction. We concentrate our study on the properties of hot nuclear matter in the density range $n_{0}<n<6 n_{0}$ (where $n_{0}=0.16$ $\mathrm{fm}^{-3}$ is the saturation density) and temperature range $0<T<30 \mathrm{MeV}$, taking into account that nuclear matter consists of neutrons, protons, electrons and muons with their relative concentrations determined from the conditions of charge neutrality and equilibrium under $\beta$-decay process in the absence of neutrino trapping.

The article is organized as follows. In Sec. II the model and relative formulas are discussed and analyzed. Results are reported and discussed in Sec. III, whereas the summary of the work is given in Sec. IV.

\section{The model}

We start by outlining the momentum dependent interaction model, then we define the thermodynamic quantities of nuclear matter and finally we analyze the $\beta$-equilibrium conditions, the contribution on pressure and energy of leptons and the total equation of state of nuclear matter.

\subsection{Momentum dependent interaction model}

The schematic potential model, employed here, is designed to reproduce the results of the microscopic calculations of both nuclear and neutron-rich matter at zero temperature and can be extended to finite temperature [2]. The energy density of the asymmetric nuclear matter (ANM) is given by the relation

$$
\epsilon\left(n_{n}, n_{p}, T\right)=\epsilon_{k i n}^{n}\left(n_{n}, T\right)+\epsilon_{k i n}^{p}\left(n_{p}, T\right)+V_{\text {int }}\left(n_{n}, n_{p}, T\right),
$$

where $n_{n}\left(n_{p}\right)$ is the neutron (proton) density and the total baryon density is $n=n_{n}+n_{p}$. The contributions of the kinetic parts are

$$
\epsilon_{k i n}^{n}\left(n_{n}, T\right)+\epsilon_{k i n}^{p}\left(n_{p}, T\right)=2 \int \frac{d^{3} k}{(2 \pi)^{3}} \frac{\hbar^{2} k^{2}}{2 m}\left(f_{n}\left(n_{n}, k, T\right)+f_{p}\left(n_{p}, k, T\right)\right),
$$

where $f_{\tau}$, (for $\left.\tau=n, p\right)$ is the Fermi-Dirac distribution function with the form

$$
f_{\tau}\left(n_{\tau}, k, T\right)=\left[1+\exp \left(\frac{e_{\tau}\left(n_{\tau}, k, T\right)-\mu_{\tau}\left(n_{\tau}, T\right)}{T}\right)\right]^{-1} .
$$


The nucleon density $n_{\tau}$ is evaluated from the following integral

$$
n_{\tau}=2 \int \frac{d^{3} k}{(2 \pi)^{3}} f_{\tau}\left(n_{\tau}, k, T\right)=2 \int \frac{d^{3} k}{(2 \pi)^{3}}\left[1+\exp \left(\frac{e_{\tau}\left(n_{\tau}, k, T\right)-\mu_{\tau}\left(n_{\tau}, T\right)}{T}\right)\right]^{-1} .
$$

In Eq. (3),$e_{\tau}\left(n_{\tau}, k, T\right)$ is the single particle energy (SPE) and $\mu_{\tau}\left(n_{\tau}, T\right)$ stands for the chemical potential of each species. The SPE has the form

$$
e_{\tau}\left(n_{\tau}, k, T\right)=\frac{\hbar^{2} k^{2}}{2 m}+U_{\tau}\left(n_{\tau}, k, T\right)
$$

where the single particle potential $U_{\tau}\left(n_{\tau}, k, T\right)$ is obtained by the functional derivative of the interaction part of the energy density with respect to the distribution function $f_{\tau}$. Including the effect of finite-range forces between nucleons, to avoid acausal behavior at high densities, the potential contribution is parameterized as follows [2]

$$
\begin{aligned}
V_{i n t}\left(n_{n}, n_{p}, T\right) & =\frac{1}{3} A n_{0}\left[\frac{3}{2}-\left(\frac{1}{2}+x_{0}\right) I^{2}\right] u^{2}+\frac{\frac{2}{3} B n_{0}\left[\frac{3}{2}-\left(\frac{1}{2}+x_{3}\right) I^{2}\right] u^{\sigma+1}}{1+\frac{2}{3} B^{\prime}\left[\frac{3}{2}-\left(\frac{1}{2}+x_{3}\right) I^{2}\right] u^{\sigma-1}} \\
& +u \sum_{i=1,2}\left[C_{i}\left(\mathcal{J}_{n}+\mathcal{J}_{p}\right)+\frac{\left(C_{i}-8 Z_{i}\right)}{5} I\left(\mathcal{J}_{n}-\mathcal{J}_{p}\right)\right],
\end{aligned}
$$

where

$$
\mathcal{J}_{\tau}=2 \int \frac{d^{3} k}{(2 \pi)^{3}} g\left(k, \Lambda_{i}\right) f_{\tau}\left(n_{\tau}, k, T\right) .
$$

In Eq. ([6) $), I$ is the asymmetry parameter $\left(I=\left(n_{n}-n_{p}\right) / n\right)$ and $u=n / n_{0}$, with $n_{0}$ denoting the equilibrium symmetric nuclear matter density, $n_{0}=0.16 \mathrm{fm}^{-3}$. The asymmetry parameter $I$ is related to the proton fraction $Y_{p}$ by the equation $I=\left(1-2 Y_{p}\right)$. The parameters $A, B, \sigma, C_{1}$, $C_{2}$ and $B^{\prime}$, which appear in the description of symmetric nuclear matter, are determined in order that $E\left(n=n_{0}\right)-m c^{2}=-16 \mathrm{MeV}, n_{0}=0.16 \mathrm{fm}^{-3}$, and the incompressibility to be $K=240$ $\mathrm{MeV}$. The additional parameters $x_{0}, x_{3}, Z_{1}$, and $Z_{2}$, which are used to determine the properties of asymmetric nuclear matter, are treated as parameters constrained by empirical knowledge [2]. The parameterizations used in the present model have only a modest microscopic foundation. Nonetheless, they have the merit of being able to closely approximate more physically motivated calculations as presented in Fig. 1. More precisely, in Fig. 1 we compare the energy per baryon (for symmetric nuclear matter (Fig. 1a) and pure neutron matter (Fig. 1b)) calculated by the present schematic model (MDIM), with those of existent, state of the art calculations by Wiringa et al. [41] and Pandharipande et al. [42].

The first two terms of the right-hand side of Eq. (6) arise from local contact nuclear interaction which lead to power density contributions as in the standard Skyrme equation of state. The first one concerns attractive interaction while the second one is repulsive, and both are assumed to be temperature independent. The third term describes the effects of finite range interactions according to the chosen function $g\left(k, \Lambda_{i}\right)$, and is the temperature dependent part of the interaction. This interaction is attractive and important at low momentum, but it weakens and disappears at very high momentum. The function $g\left(k, \Lambda_{i}\right)$, suitably chosen to simulate finite range effects, has the following form [2]

$$
g(k, \Lambda)=\left[1+\left(\frac{k}{\Lambda_{i}}\right)^{2}\right]^{-1}
$$

where the finite range parameters are $\Lambda_{1}=1.5 k_{F}^{0}$ and $\Lambda_{2}=3 k_{F}^{0}$ and $k_{F}^{0}$ is the Fermi momentum at the saturation point $n_{0}$. 
The main origin of the momentum dependence in Brueckner theory is the nonlocality of the exchange interaction. Following the discussion of Bertsch et al. [43] a single-particle potential $U(n)$ which depends only on the baryon density is oversimplified. What is more, it is well known that nuclear interaction has strong exchange effects which give rise to a momentum dependence in the single-particle potential and as a consequence has an effect on the energy density functional. The question here is how best to parameterize the momentum dependence in modelling the potential $U(n, k)$. A promising approach might be to adopt the relativistic mean field model, where $U(n, k)=U_{\nu} n+\frac{U_{s} n}{\sqrt{1+k^{2} / m^{2}}}$. The above potential exhibits a strong momentum dependence for small $k$ which diminishes to zero at high momentum. In order to perform extensive studies in heavy ion collision studies, Gale et al. 44] have proposed the following parametrization for the momentum part of the single-particle

$$
U(n, k) \sim C \frac{n}{n_{0}} \frac{1}{1+\left(\mathbf{k}-\left\langle\mathbf{k}^{\prime}\right\rangle\right)^{2} / \Lambda^{2}} .
$$

This has a proper fall-off at high $k$ and Galilean invariance is assured by measuring $k$ with respect to the average of the particles in the neighborhood, $\left\langle\mathbf{k}^{\prime}\right\rangle$. For static nuclear matter we have $\left\langle\mathbf{k}^{\prime}\right\rangle=0$.

The present model, which is a generalization of that proposed by Gale et al. [44, has been successfully applied in heavy ion collisions and astrophysical studies over the years [2, 3, 4, 27, 28, 33, 45, 46.

In order to clarify the relative contribution of the three terms of the potential energy density mentioned above, we plot them as a function of the baryon density in Fig. 2a. In this figure we have that

$$
\begin{aligned}
V_{A} & =\frac{1}{3} A n_{0}\left[\frac{3}{2}-\left(\frac{1}{2}+x_{0}\right) I^{2}\right] u^{2} \\
V_{B} & =\frac{\frac{2}{3} B n_{0}\left[\frac{3}{2}-\left(\frac{1}{2}+x_{3}\right) I^{2}\right] u^{\sigma+1}}{1+\frac{2}{3} B^{\prime}\left[\frac{3}{2}-\left(\frac{1}{2}+x_{3}\right) I^{2}\right] u^{\sigma-1}} \\
V_{C} & =u \sum_{i=1,2}\left[C_{i}\left(\mathcal{J}_{n}^{i}+\mathcal{J}_{p}^{i}\right)+\frac{\left(C_{i}-8 Z_{i}\right)}{5} I\left(\mathcal{J}_{n}^{i}-\mathcal{J}_{p}^{i}\right)\right] .
\end{aligned}
$$

As mentioned above, the first term $V_{A}$ corresponds to an attractive interaction, whereas the second one $V_{B}$ corresponds to repulsive interaction and dominates for high values of $n\left(n>0.6 \mathrm{fm}^{-3}\right)$. Both of these terms are temperature independent. The third term $V_{C}$ (is plotted for $T=0$ ) contains the momentum dependent part of the interaction, corresponds to attractive interaction, and its main contribution is to compete with the repulsive interaction of $V_{B}$ for high values of $n$ and as a consequence avoid acausal behavior of the EOS at high densities. The term $V_{C}$ consists of two finite range terms, one corresponding to a long-range attraction and the other to a short-range repulsion.

Thermal effects on the momentum dependent term $V_{C}$ are displayed in Fig. $2 \mathrm{~b}$. The contribution of $V_{C}$ is plotted for various values of $T$. It is therefore concluded that thermal effects are more pronounced for high values of $T(T>10 \mathrm{MeV})$, leading to a less attractive contribution. More precisely, we find that for small values of $n$ (i.e. $n=0.15 \mathrm{fm}^{-3}$ ) $V_{C}$ increases (compared to the cold case $T=0) 3 \%-20 \%$ for $T=10-30$. For higher values of $n$ the increase is even less.

An additional test for the present model is to compare the single particle potential $U_{\tau}\left(n_{\tau}, k, T\right)$ (or $U_{\tau}(n, I, k, T)$ ) originated from the present version of the momentum dependent interaction with other calculations. The single particle potential $U_{\tau}(n, I, k, T)$ (protons or neutrons), obtained from the functional derivative of the interaction part of the energy density (Eq. (6) ) with respect to the 
distribution function $f_{\tau}$, has the general form [40]

$$
U_{\tau}(n, I, k, T)=U_{\tau}^{A}(n, I)+U_{\tau}^{B}(n, I)+U_{\tau}^{M D}(n, I, k, T) .
$$

It is of interest to see that the single particle potentials are separated into two parts. The first one, $U_{\tau}^{A}(n, I)+U_{\tau}^{B}(n, I)$ is a function only of the baryon density $n$ and the isospin asymmetry parameter $I$. The second one, $U_{\tau}^{M D}(n, I, k, T)$ has an additional dependence on $T$ and $k$. Actually, $U_{\tau}^{M D}(n, I, k, T)$ is mainly responsible for the trend of the effective mass and also the effective mass splitting. Additionally, it is connected with the effect of the temperature on the interacting part of the energy density [40].

The single-particle potential in symmetric nuclear matter has been calculated microscopically for several Hamiltonians by Wiringa [47]. These Hamiltonians include nucleon-nucleon potentials fit to scattering data and three nucleon potentials fit to binding energies of few-body nuclei and saturation properties of nuclear matter. The potential was parameterized using the ansatz

$$
U(n, k)=\alpha(n)+\frac{\beta(n)}{1+\left(\frac{k}{\Lambda(n)}\right)^{2}},
$$

where the density dependent parameters $\alpha(n), \beta(n)$ and $\Lambda(n)$, for three types Hamiltonians, are listed in Table I of Ref. [47.

Furthermore, the single-particle potential has been derived by Li et al. [48]. The derivation is based upon the Bonn meson-exchange model for the nucleon-nucleon interaction and the DiracBrueckner approach for nuclear matter. The potential, named DBHF, has been parameterized as following

$$
U(n, k)=\alpha n+\beta n^{\gamma}+\delta \ln ^{2}\left(\epsilon(\hbar c k)^{2}+1\right) n^{\sigma} .
$$

The parameters $\alpha, \beta, \gamma, \delta, \epsilon$ and $\sigma$ are listed in Table I of Ref. [48].

A comparison with the results of UV14+TNI, UV14+UVII, AV14+UVII and DBHF interactions show that (see Fig. 3) $U(n, k)$ (for $T=0$ ) obtained from the present model is very reasonable for at least up to the value $k=3 \mathrm{fm}^{-1}$. The agreement is not so good for high values of $k$, especially compared with the DBHF interaction, but as has been pointed out by $\mathrm{Li}$ et al. [48, the parametrization in Eq. 12 is bad for large $k$ since it continues to grow with increasing $k$, while the exact potential becomes independent of $k$ for large momenta. In conclusion, the present results show that the momentum dependent interaction model, which has been applied in the present work, provides a reliable representation of $U(n, k)$ for a wide range of density and momentum.

The energy density of asymmetric nuclear matter at density $n$ and temperature $T$, in a good approximation, is expressed as

$$
\epsilon(n, T, I)=\epsilon(n, T, I=0)+\epsilon_{\text {sym }}(n, T, I),
$$

where

$$
\epsilon_{\text {sym }}(n, T, I)=n I^{2} E_{\text {sym }}^{\text {tot }}(n, T)=n I^{2}\left(E_{\text {sym }}^{\text {kin }}(n, T)+E_{\text {sym }}^{\text {int }}(n, T)\right) .
$$

In Eq. (14) the nuclear symmetry energy $E_{\text {sym }}^{\text {tot }}(n, T)$ is separated in two parts corresponding to the kinetic contribution $E_{s y m}^{k i n}(n, T)$ and the interaction one $E_{s y m}^{i n t}(n, T)$.

From Eqs. (13) and (14) and setting $I=1$, we find that the nuclear symmetry energy $E_{\text {sym }}^{\text {tot }}(n, T)$ is given by

$$
E_{\text {sym }}^{\text {tot }}(n, T)=\frac{1}{n}(\epsilon(n, T, I=1)-\epsilon(n, T, I=0)) .
$$

Thus, from Eq. (15) and by a suitable choice of the parameters $x_{0}, x_{3}, Z_{1}$ and $Z_{2}$, we can obtain various forms for the density dependence of the symmetry energy $E_{\text {sym }}^{\text {tot }}(n, T)$. 
It is well known that the need to explore different forms for $E_{\text {sym }}^{\text {tot }}(n, T)$ stems from the uncertain behavior at high density [2]. The high-density behavior of symmetry energy is the least known property of dense matter [49, 50, 51], with different nuclear models giving contradictory predictions. Thus, in relativistic mean field (RMF) models, the symmetry energy increases strongly with the density of nuclear matter [52, while in many realistic potential models of nuclear matter in the variational approach [7, 53], the symmetry energy saturates and then bends over at higher densities.

Recently, the density dependence of the symmetry energy in the equation of state of isospin asymmetric nuclear matter has been studied using isoscaling of the fragment yields and the antisymmetrized molecular dynamic calculation [54]. It was observed that the experimental data at low densities are consistent with the form of symmetry energy, $E_{\text {sym }}(u) \approx 31.6 u^{0.69}$, in close agreement with those predicted by the results of variational many-body calculations. In Ref. [54] it was suggested also that the heavy ion studies favor a dependence of the form $E_{\text {sym }}(u) \approx 31.6 u^{\gamma}$, where $\gamma=0.6-1.05$. This constrains the form of the density dependence of the symmetry energy at higher densities, ruling out an extremely "stiff" and "soft" dependence [54].

Additionally, Chen et al. [55] also showed, using the isospin dependent Boltzmann-UehlingUhlenbeck transport model calculations, that a stiff density dependence of the symmetry energy parameterized as, $E_{\text {sym }}(u) \approx 31.6 u^{1.05}$ explains well the isospin diffusion data [56] from NSCLMSU (National Superconducting Cyclotron Laboratory at Michigan State University).

In this paper, since we are interested mainly in the study of thermal effects on the nuclear symmetry energy, we choose a specific form for it, enabling us to reproduce accurately the results of many other theoretical studies [57, 58]. In Ref. [57] the authors carried out a systematic analysis of the nuclear symmetry energy in the formalism of the relativistic Dirac-Brueckner-Hartree-Fock approach, using the Bonn one-boson-exchange potential. In a very recent work [58], the authors applied a similar method as in Ref. [57] for the microscopic predictions of the equation of state in asymmetric nuclear matter. In that case $E_{\text {sym }}(u)$ is obtained employing the simple parametrization $E_{\text {sym }}(u)=C u^{\gamma}$ with $\gamma=0.7-1.0$ and $C \approx 32 \mathrm{MeV}$. The authors conclude that a value of $\gamma$ close to 0.8 gives a reasonable description of their predictions, although the use of different functions in different density regions may be best for an optimal fit [58]. The results of Refs. [57, 58] are well reproduced by parameterizing the nuclear symmetry energy according to the formula

$$
E_{\text {sym }}^{\text {tot }}(n, T=0)=\underbrace{13 u^{2 / 3}}_{\text {Kinetic }}+\underbrace{17 F(u)}_{\text {Interaction }} \text {. }
$$

For the function $F(u)$, which parametrizes the interaction part of the symmetry energy, we apply the following form

$$
F(u)=u \text {. }
$$

The parameters $x_{0}, x_{3}, Z_{1}$ and $Z_{2}$ are chosen so that Eq. (15), for $T=0$, reproduces the results of Eq. (16) for the function $F(u)=u$.

In one of our previous paper [59], the potential part of the symmetry energy has been parameterized in the generalized form, $F(u)=u^{c}$, and the obtained nuclear equations of state are applied to the systematic study of the global properties of a neutron star (masses, radii and composition). We obtained a linear relation between the parameter $c$ and the radius and the maximum mass of the neutron star [59]. Additionally, we found that a linear relation between the radius and the derivative of the symmetry energy near the saturation density $n_{0}$ holds [59].

It is worthwhile to point out that the above parametrization of the interacting part of the nuclear symmetry energy is used extensively for the study of neutron star properties [2, 60], as well as for the study of the collisions of neutron-rich heavy ions at intermediate energies [61, 62]. For a very recent review of the applications of the proposed momentum dependent effective interaction model and its specific parameterizations, see Ref. [4] (and references therein). 


\subsection{Thermodynamic description of hot nuclear matter}

In order to study the properties of nuclear matter at finite temperature, we need to introduce the Helmholtz free energy $F$. The differential of the total free energy $F_{\text {tot }}$ (the total free energy of baryons contained in volume $V$ ) and total internal energy $E_{t o t}$ (the total internal energy of baryons contained in volume $V$ ) are given by [63, 64]

$$
\begin{gathered}
\mathrm{d} F_{t o t}=-S_{t o t} \mathrm{~d} T-P \mathrm{~d} V+\sum_{i} \mu_{i} \mathrm{~d} N_{i} \\
\mathrm{~d} E_{t o t}=T \mathrm{~d} S_{t o t}-P \mathrm{~d} V+\sum_{i} \mu_{i} \mathrm{~d} N_{i}
\end{gathered}
$$

where $S_{t o t}$ is the total entropy of the baryons, while $\mu_{i}$ and $N_{i}$ are the chemical potential and the number of particles of each species respectively.

It is easy to prove that the free energy per particle $F$ is written as [63, 64]

$$
F(n, T, I)=E(n, T, I)-T S(n, I, T) .
$$

In Eq. (20), $E$ is the internal energy per particle, $E=\epsilon / n$, and $S$ is the entropy per particle, $S=s / n$. From Eq. (20) is also concluded that for $T=0$, the free energy $F$ and the internal energy $E$ coincide.

The entropy density $s$ has the same functional form as that of a non interacting gas system, given by the equation

$$
s_{\tau}(n, I, T)=-2 \int \frac{d^{3} k}{(2 \pi)^{3}}\left[f_{\tau} \ln f_{\tau}+\left(1-f_{\tau}\right) \ln \left(1-f_{\tau}\right)\right] .
$$

The total internal energy $E_{\text {tot }}$ is useful for studying isentropic processes. In that description of a thermodynamic system, the pressure and the chemical potential are defined as follows [63, 64]

$$
P=-\left(\frac{\partial E_{t o t}}{\partial V}\right)_{S, N_{i}}=n^{2}\left(\frac{\partial \epsilon / n}{\partial n}\right)_{S, N_{i}}, \quad \quad \mu_{i}=\left(\frac{\partial E_{t o t}}{\partial N_{i}}\right)_{S, V, N_{j \neq i}}=\left(\frac{\partial \epsilon}{\partial n_{i}}\right)_{S, V, n_{j \neq i}} .
$$

Now we are going to study the properties and the EOS of nuclear matter by considering an isothermal process. In that, the pressure and the chemical potential are connected with the derivative of the total free energy $F_{t o t}$. More specifically, they are defined as follows

$$
P=-\left(\frac{\partial F_{t o t}}{\partial V}\right)_{T, N_{i}}=n^{2}\left(\frac{\partial f / n}{\partial n}\right)_{T, N_{i}}, \quad \quad \mu_{i}=\left(\frac{\partial F_{t o t}}{\partial N_{i}}\right)_{T, V, N_{j \neq i}}=\left(\frac{\partial f}{\partial n_{i}}\right)_{T, V, n_{j \neq i}},
$$

where $f$ is the free energy density.

The pressure $P$ can be calculated also from equations [63, 64]

$$
V P=T S_{t o t}-E_{t o t}+\sum_{i} \mu_{i} N_{i}, \quad \text { or } \quad P=T s-\epsilon+\sum_{i} \mu_{i} n_{i}
$$

It is also possible to calculate the entropy per particle $S(n, T)$ by differentiating the free energy density $f$ with respect to the temperature

$$
S(n, T)=-\left(\frac{\partial f / n}{\partial T}\right)_{V, N_{i}}
$$

The comparison of the two entropies, that is from Eqs. (21) and (25), provides a test of the approximation used in the present work. 
It is easy to show by applying Eq. (23) that (see for a proof [60] as well as [32])

$$
\begin{aligned}
\mu_{n} & =F+u\left(\frac{\partial F}{\partial u}\right)_{Y_{p}, T}-Y_{p}\left(\frac{\partial F}{\partial Y_{p}}\right)_{n, T}, \\
\mu_{p} & =\mu_{n}+\left(\frac{\partial F}{\partial Y_{p}}\right)_{n, T}, \\
\hat{\mu} & =\mu_{n}-\mu_{p}=-\left(\frac{\partial F}{\partial Y_{p}}\right)_{n, T} .
\end{aligned}
$$

We can define the symmetry free energy per particle $F_{\text {sym }}(n, T)$ by the following parabolic approximation (see also [32, 33])

$$
F(n, T, I)=F(n, T, I=0)+I^{2} F_{\text {sym }}(n, T)=F(n, T, I=0)+\left(1-2 Y_{p}\right)^{2} F_{\text {sym }}(n, T),
$$

where

$$
F_{\text {sym }}(n, T)=F(n, T, I=1)-F(n, T, I=0) .
$$

It is worthwhile to notice that the above approximation is not valid from the beginning, but one needs to check the validity of the parabolic law in the present model before using it. As we see later, that law is well satisfied as well as the parabolic law holding for the energy.

Now, by applying Eq. (27) in Eq. (26), we obtain the key relation

$$
\hat{\mu}=\mu_{n}-\mu_{p}=4\left(1-2 Y_{p}\right) F_{s y m}(n, T) .
$$

The above equation is similar to that obtained for cold nuclear matter by replacing $E_{\text {sym }}(n)$ with $F_{\text {sym }}(n, T)$.

\section{$2.3 \quad \beta$-equilibrium, leptons contribution and equation of state}

Stable high density nuclear matter must be in chemical equilibrium for all types of reactions, including the weak interactions, while $\beta$ decay and electron capture take place simultaneously

$$
n \longrightarrow p+e^{-}+\bar{\nu}_{e}, \quad p+e^{-} \longrightarrow n+\nu_{e}
$$

Both types of reactions change the electron per nucleon fraction, $Y_{e}$ and thus affect the equation of state. Here, we assume that neutrinos generated in those reactions have left the system. The absence of neutrino-trapping has a dramatic effect on the equation of state and mainly induces a significant change on the values of the proton fraction $Y_{p}$ [9, 10]. The absence of neutrinos implies that

$$
\hat{\mu}=\mu_{n}-\mu_{p}=\mu_{e} .
$$

When the energy of electrons is large enough (i.e. greater than the muon mass), it is energetically favorable for the electrons to convert to muons

$$
e^{-} \longrightarrow \mu^{-}+\bar{\nu}_{\mu}+\nu_{e}
$$

Denoting the muon chemical potential by $\mu_{\mu}$, the chemical equilibrium established by the above process and its inverse is given by

$$
\mu_{e}=\mu_{\mu}
$$

Taking into account that the threshold for muons occurs for $\mu_{\mu}=m_{\mu} c^{2} \simeq 105.7 \mathrm{MeV}$, one may expect muons to appear roughly at nuclear density $n=0.16 \mathrm{fm}^{-3}$. 
Thus, in total, we consider that nuclear matter contains neutrons, protons, electrons, and muons. They are in a $\beta$-equilibrium, where the following relations hold

$$
\mu_{n}=\mu_{p}+\mu_{e}, \quad \mu_{e}=\mu_{\mu}
$$

Furthermore, they obey the charge neutrality condition i.e.

$$
n_{p}=n_{e}+n_{\mu}
$$

The leptons (electrons and muons) density is given by the expression

$$
n_{l}=\frac{2}{(2 \pi)^{3}} \int \frac{\mathrm{d} \mathbf{k}}{1+\exp \left[\frac{\sqrt{\hbar^{2} k^{2} c^{2}+m_{l}^{2} c^{4}}-\mu_{l}}{T}\right]} .
$$

One can solve self-consistently Eqs. (29), (33), (34) and (35) in order to calculate the proton fraction $Y_{p}$, the lepton fractions $Y_{e}$ and $Y_{\mu}$, as well as the electron chemical potential $\mu_{e}$ as a function of the baryon density $n$, for various values of the temperature $T$.

The next step is to calculate the energy and pressure of leptons given by the following formulae

$$
\begin{gathered}
\epsilon_{l}\left(n_{l}, T\right)=\frac{2}{(2 \pi)^{3}} \int \frac{\sqrt{\hbar^{2} k^{2} c^{2}+m_{l}^{2} c^{4}} \mathrm{~d} \mathbf{k}}{1+\exp \left[\frac{\sqrt{\hbar^{2} k^{2} c^{2}+m_{l}^{2} c^{4}}-\mu_{l}}{T}\right]} \\
P_{l}\left(n_{l}, T\right)=\frac{1}{3} \frac{2(\hbar c)^{2}}{(2 \pi)^{3}} \int \frac{1}{\sqrt{\hbar^{2} k^{2} c^{2}+m_{l}^{2} c^{4}}} \frac{k^{2} \mathrm{~d} \mathbf{k}}{1+\exp \left[\frac{\sqrt{\hbar^{2} k^{2} c^{2}+m_{l}^{2} c^{4}}-\mu_{l}}{T}\right]} .
\end{gathered}
$$

The chemical potentials of electrons and muons are equal and according to Eqs. (29) and (33) are

$$
\mu_{e}=\mu_{\mu}=\mu_{p}-\mu_{n}=4\left(1-2 Y_{p}(n, T)\right) F_{\text {sym }}(n, T)=4 I(n, T) F_{\text {sym }}(n, T) .
$$

The equation of state of hot nuclear matter in $\beta$-equilibrium (considering that it consists of neutrons, protons, electrons and muons) can be obtained by calculating the total energy density $\epsilon_{t o t}$ as well as the total pressure $P_{t o t}$. The total energy density is given by

$$
\epsilon_{t o t}(n, T, I)=\epsilon_{b}(n, T, I)+\sum_{l=e, \mu} \epsilon_{l}(n, T, I)
$$

where $\epsilon_{b}(n, T, I)$ and $\epsilon_{l}(n, T, I)$ are the contributions of baryons and leptons respectively. The total pressure is

$$
P_{t o t}(n, T, I)=P_{b}(n, T, I)+\sum_{l=e, \mu} P_{l}(n, T, I),
$$

where $P_{b}(n, T, I)$ is the contribution of the baryons (see Eq. (24)) i.e.

$$
P_{b}(n, T, I)=T \sum_{\tau=p, n} s_{\tau}(n, T, I)+\sum_{\tau=n, p} n_{\tau} \mu_{\tau}(n, T, I)-\epsilon_{b}(n, T, I),
$$

while $P_{l}(n, T, I)$ is the contribution of the leptons (see Eq. (37)). From Eqs. (39) and (40) we can construct the isothermal curves for energy and pressure and finally derive the isothermal behavior of the equation of state of hot nuclear matter under $\beta$-equilibrium. 


\section{Results and Discussion}

The schematic potential model, which has been applied in the present work, has been designed to reproduce the results of the more microscopic calculations of both nuclear and neutron-rich matter up to high values of baryon density (see Fig. 1). The behavior of the high density EOS is of great significance to the determination of hot protoneutron stars and cold neutron stars structure. The model has the additional advantage that with the appropriate parametrization, is able to reproduce different forms of the density dependence of the nuclear symmetry energy.

In view of the above discussion we calculate the equation of state of hot asymmetric nuclear matter by applying a momentum dependent effective interaction model describing the baryons interaction. We consider that nuclear matter contains neutrons, protons, electrons and muons under $\beta$-equilibrium and charge neutrality. The key quantities in our calculations are the proton fraction $Y_{p}$ and also the asymmetry free energy defined in Eq. (28).

In order to check the validity of the parabolic approximation (27), we plot in Fig. 4 the difference $F(n, T, I=1)-F(n, T, I=0)$ as a function of $\left(1-2 Y_{p}\right)^{2}$ at temperature $T=10$ and $T=30 \mathrm{MeV}$ for three baryon densities, i.e., $n=0.2 \mathrm{fm}^{-3}, n=0.3 \mathrm{fm}^{-3}$, and $n=0.4$ $\mathrm{fm}^{-3}$. It is seen that in a good approximation an almost linear relation holds between $F(n, T, I=$ $1)-F(n, T, I=0)$ and $\left(1-2 Y_{p}\right)^{2}$. A similar behavior of $F_{s y m}(n, T)$ is found by $\mathrm{Xu}$ et al. [33], applying an isospin and momentum dependent interaction model.

It is worth to present the calculation recipe of our work. The outline of our approach is the following: For a fixed baryon density $n$, temperature $T$, and asymmetry parameter $I$, Eq. (44) may be solved iteratively in order to calculate the quantity

$$
\eta_{\tau}\left(n_{\tau}, T\right)=\frac{\mu_{\tau}\left(n_{\tau}, T\right)-\tilde{U}_{\tau}\left(n_{\tau}, T\right)}{T},
$$

where

$$
\tilde{U}_{\tau}\left(n_{\tau}, T\right)=U_{\tau}\left(n_{\tau}, k, T\right)-\tilde{U}_{\tau}\left(n_{\tau}, k\right) .
$$

Knowledge of $\eta_{\tau}(n, T)$ allows the evaluation of $\tilde{U}_{\tau}\left(n_{\tau}, T\right)$, which then may be employed to infer the chemical potential from

$$
\mu_{\tau}\left(n_{\tau}, T\right)=T \eta_{\tau}\left(n_{\tau}, T\right)+\tilde{U}\left(n_{\tau}, T\right)
$$

required as an input for the calculation of the Fermi-Dirac distribution function $f_{\tau}\left(n_{\tau}, k, T\right)$. The knowledge of $f_{\tau}\left(n_{\tau}, k, T\right)$ permits the calculation of the bulk quantities of asymmetric nuclear matter.

$F_{\text {sym }}(n, T)$, for various values of the temperature $T$, was derived with a least-squares fit to the numerical values according to Eq. (28) and has the form

$$
\begin{aligned}
F_{\text {sym }}(u ; T=0) & =13 u^{2 / 3}+17 u \\
F_{\text {sym }}(u ; T=5) & =3.653+28.018 u-1.512 u^{2}+0.185 u^{3}-0.001 u^{4}, \\
F_{\text {sym }}(u ; T=10) & =5.995+26.157 u-0.827 u^{2}+0.068 u^{3}-0.002 u^{4}, \\
F_{\text {sym }}(u ; T=20) & =13.200+21.267 u+0.800 u^{2}-0.193 u^{3}+0.014 u^{4}, \\
F_{\text {sym }}(u ; T=30) & =21.087+17.626 u+1.645 u^{2}-0.289 u^{3}+0.018 u^{4} .
\end{aligned}
$$

where the case with $T=0$, is included as well. In that case $F_{\text {sym }}$ coincides with $E_{\text {sym }}$.

In Fig. 5 we present the behavior of the free energy, corresponding to hot $\beta$-stable nuclear matter, as a function of the baryon density $n$, for various values of the temperature $T$. It is obvious that the thermal effects are more pronounced for low values of the density $n$. 
In Fig. 6 we plot the calculated free energy for symmetric nuclear matter and pure neutron matter of the proposed momentum dependent interaction model in comparison with the values of the free energy calculated by Friedman and Pandharipande (FP model) [7]. In the FP model the equation of state of hot and cold nuclear and neutron matter has been calculated in the framework of a variational calculation, where a realistic nuclear interaction containing two- and three-nucleon body nucleon-nucleon interaction has been used. In the case of symmetric nuclear matter the results of the two models are very similar up to values $n=0.4-0.5 \mathrm{fm}^{-3}$ depending on the values of $T$. The above agreement is expected, in the sense that a part of the parameters of the model applied in the present work are determined from constraints provided by the empirical properties of symmetric nuclear matter at the equilibrium density $n_{0}=0.16 \mathrm{fm}^{-3}$.

However, there is an obvious disagreement in the case of pure nuclear matter, where in the two models the free energy exhibits a different trend, especially for higher values of $n$. The above disagreement will be explained below.

In Fig. 7 we display the internal energy per particle $E(n, T)=\epsilon(n, T) / n$ given by Eq. (11) for various values of temperature. Thermal effects, as expected, are more pronounced for low values of the baryon density $n$ and less important for high values of $n$.

In Fig. 8 we display the internal energy of symmetric nuclear matter and pure neutron matter, for $T=0$, calculated by the MDIM and FP models. In accordance with the case of the free energy, there is a very good agreement in symmetric nuclear matter, but an obvious disagreement is exhibited in pure neutron matter. The explanation of the agreement in the first case is the same as in the case of the free energy. The disagreement is due to the completely different behavior of the two models of the nuclear symmetry energy, presented in Fig. 9. In our model the parameters $x_{0}, x_{3}, Z_{1}$ and $Z_{2}$ chosen so that Eq. (15), for $T=0$, reproduce the results of Eq. (16) for the function $F(u)=u$. Consequently, $E_{\text {sym }}(n)$ shows an increasing trend shown in Fig. 9. In contrast, in the FP model, $E_{\text {sym }}(n)$ is a slightly increasing function of $n$ for low $n$ and then a decreasing function of $n$ for $n>0.5 \mathrm{fm}^{-3}$.

In addition, we plot the nuclear symmetry energy extracted from experimental results and presented in Ref. [54], where $E_{\text {sym }}(u)$ is parameterized according to the relation $E_{\text {sym }}(u) \approx 31.6 u^{0.69}$ as well as experimental results extracted from Ref. [55], where $E_{\text {sym }}(u)$ is given by $E_{\text {sym }}(u) \approx$ $31.6 u^{1.05}$. The important point to be noted is that both cases clearly favor a stiff density dependence of the symmetry energy at higher densities, ruling out the very stiff and very soft predictions. These results can thus be employed to constrain the form of the density dependence of the symmetry energy at supranormal densities relevant for neutron star studies [54]. In the same figure the theoretical predictions of Ref. [58] are presented, where $E_{\text {sym }}(u)$ is parameterized by $E_{\text {sym }}(u) \approx 32 u^{0.8}$.

The results of Ref. [54] are in a good agrement with the present model up to $n=0.3 \mathrm{fm}^{-3}$ while the theoretical predictions of Ref. [58] are very close to the present model up to very high values of the baryon density $n$.

However, our motivation, here, is not to perform a systematic comparison of various models, but we would like just to present the similarities and the deviations existing between the models. The deviations, concerning the symmetry energy behavior of the two models (MDIM and FP model) are well reflected on the behavior of the free energy and internal energy of pure neutron matter as shown in Figs. 6 and 8.

In Fig. 10 we plot the thermal energy per particle

$$
E_{\text {thermal }}(n, T)=E(n, T)-E(n, T=0),
$$

of $\beta$-stable matter as a function of the baryon density $n$ for various values of temperature $T$. The most striking feature of $E_{\text {thermal }}(n, T)$ is that for small values of $T$, the thermal contribution to 
the internal energy is almost independent of the density $n$. For high values of $T$ the situation is different and $E_{\text {thermal }}(n, T)$, for fixed values of $T$, is a decreasing function of the density $n$.

$E_{\text {thermal }}(n, T)$ can be decomposed to separate contributions of the kinetic and potential energies as follows:

$$
E_{\text {thermal }}(n, T)=E_{\text {thermal }}^{\text {kin }}(n, T)+E_{\text {thermal }}^{\text {pot }}(n, T) .
$$

We find that for small values of the baryon density (i.e. $n=0.2 \mathrm{fm}^{-3}$ ) the contribution, to $E_{\text {thermal }}(n, T)$ of the potential energy $E_{\text {thermal }}^{\text {pot }}(n, T)$ is $20 \%-10 \%$ for $T=5-30 \mathrm{MeV}$. For medium values of $n$ (i.e. $n=0.4 \mathrm{fm}^{-3}$ ) is $43 \%-20 \%$ for $T=5-30 \mathrm{MeV}$ and for higher values (i.e. $n=0.6 \mathrm{fm}^{-3}$ ) is $70 \%-30 \%$ for $T=5-30 \mathrm{MeV}$. Hence, it is concluded that the potential part of the energy (as a result of the momentum dependence of the interaction) contributes significantly to the thermal energy, mainly for small values of $T$ (for fixed values of $n$ ) and for large values of $n$ (for fixed values of $T$ ).

At this point, it is worthwhile to compare the results for the pressure obtained by applying Eqs. (24) and (23). Thus, in Fig. 11 we plot $P$ of asymmetric nuclear matter for $Y_{p}=0.1$ and 0.3 at $T=10$ and $30 \mathrm{MeV}$. The full lines give the results calculated from Eq. (24), while the squares give results obtained by differentiating $F(n, T)$ (Eq. (23) ). The two calculations for the pressure are in excellent agreement. This agreement provides a test of the calculations performed in the present model.

It is of interest also to study the effect of the temperature on the baryon pressure defined by equation (24). A related quantity is the thermal pressure $P_{\text {thermal }}(n, T)$ defined as:

$$
P_{\text {thermal }}(n, T)=P(n, T)-P(n, T=0) .
$$

$P_{\text {thermal }}(n, T)$ as a function of $n$, for various values of $T$ is seen in Fig. 12. $P_{\text {thermal }}(n, T)$, in all of the cases, is an increasing function of the baryon density.

The proton fraction affects the reaction rate of neutrino process inside that star. If a neutron star has a large proton fraction, the cooling rate may drastically change through the high neutrino emissivity due to the direct Urca process. This process can occur if the proton fraction in the matter of a cold neutron star exceeds the critical value of $0.11-0.15$ and would lead to the rapid cooling of the neutron star. Thus, it is important to calculate the proton fraction as a function of the baryon density and investigate the temperature effects on that.

Fig. 13 displays the fractions of protons, electrons and muons as functions of the density, for various values of $T$. The proton fraction is an increasing function of $T$ and this effect is more pronounced for $T>10 \mathrm{MeV}$. The proton fraction $Y_{p}$ was derived also with a least-squares fit to the numerical results obtained from our calculations, leading to the following relations (for $n>0.15$ $\left.\mathrm{fm}^{-3}\right)$.

$$
\begin{aligned}
Y_{p}(n ; T=0) & =-0.050+0.633 n-0.521 n^{2}+0.184 n^{3} \\
Y_{p}(n ; T=5) & =-0.046+0.625 n-0.514 n^{2}+0.179 n^{3} \\
Y_{p}(n ; T=10) & =-0.032+0.570 n-0.436 n^{2}+0.139 n^{3} \\
Y_{p}(n ; T=20) & =0.021+0.378 n-0.163 n^{2}+0.004 n^{3} \\
Y_{p}(n ; T=30) & =0.109-0.062 n+0.908 n^{2}-1.270 n^{3}+0.580 n^{4} .
\end{aligned}
$$

In Fig. 14 we plot the Fermi distribution function $f_{p, n}(n, T)$ both for neutrons and protons for various values of $T$. We observe that the diffuseness of $f_{p}(n, T)$ is larger than that of $f_{n}(n, T)$. We give an explanation (see also [9]): the ratio of $T$ to the Fermi kinetic energy $\epsilon_{F i}$ is a measure of the thermal effect. Thus by comparing the two ratios we have (see also Appendix)

$$
\left(\frac{\left(T / \epsilon_{F p}\right)}{\left(T / \epsilon_{F n}\right)}\right)=k_{F}^{n 2} / k_{F}^{p 2}=Y_{n}^{3 / 2} / Y_{p}^{3 / 2} .
$$


But, due to $Y_{n}>Y_{p}$, we conclude that we expect the diffuseness to be larger for the proton distribution than for the neutron one, depending of course on the specific value of the ratio $Y_{p} / Y_{n}$. As we will see later, this fact influences the values of the contributions of protons and neutrons to the total entropy per particle. The entropy, according to relation (21), is an increasing function of the diffuseness. Thus, the contribution of each species on the total value of the entropy depends strongly on the diffuseness of the corresponding Fermi distribution function.

We can provide a second test, concerning the accuracy of the present calculations, by comparing the entropy per baryon calculated by applying Eqs. (21) and (25). Thus, in Fig. 15 we plot $S$ of asymmetric nuclear matter with $Y_{p}=0.2$ at $T=10,20,30 \mathrm{MeV}$. The full lines give the entropy calculated from Eq. (21), while the squares give results obtained by differentiating $F(n, T)$ with respect to $T$ (Eq. (25) ). It is obvious that there is again a very good agreement of the results, especially for low values of $T$ and high values of $n$.

In Fig. 16 we plot the contribution of the proton $S_{p}$, the neutron $S_{n}$ and the total entropy per baryon $S$. It is obvious that there is a strong effect of $T$ on the values of the entropies mainly for low values of the density. The main part of the contribution comes from neutrons, whereas the contribution of protons is three times less. It is worthwhile to notice that, in spite of $Y_{p} \sim(1 / 20-3 / 10) Y_{n}$, the approximate relation $S_{p} \sim(1 / 4-3 / 7) S_{n}$ holds. This feature is understood by the previous discussion that $f_{p}(n, T)$ is diffused more broadly than $f_{n}(n, T)$, so the larger the diffuseness, the larger is the entropy contribution (see also [9]).

In Fig. 17 we plot the contribution of the electronic $S_{e}$, the muonic $S_{\mu}$ and the total (leptonic) $S_{l}$ to the entropy per baryon. The contribution to the entropy, of $S_{e}$ depends slightly on the density, for fixed values of $T$. Our present results are very close to those found by Onsi et al. [19], where they employed the analytical approximate formula for the electron entropy density $s_{e}$

$$
s_{e}=\frac{1}{3} \frac{\mu_{e}^{2}}{(\hbar c)^{3}} T, \quad \quad \mu_{e}=\hbar c\left(3 \pi^{2} Y_{e} n\right)^{1 / 3}
$$

According to the above formula, the contribution of electrons to the entropy per baryon has the form

$$
S_{e}=s_{e} / n \sim\left(\frac{Y_{e}^{2}}{n}\right)^{1 / 3} T .
$$

The quantity $\left(\frac{Y_{e}^{2}}{n}\right)^{1 / 3}$ is a function slightly dependent on the density $n$, so that for a fixed value of $T$ the contribution $S_{e}$ is almost constant. The muonic contribution to the entropy, for fixed $T$, increases slightly as a function of the density.

In Fig. 18 we present the EOS of the $\beta$-stable hot nuclear matter by taking into account and analyzing the contribution to the total pressure of each component. The main contribution to the total pressure originates from the baryons, while the contribution of the leptons is about a few percent compared to $P_{b}$. It is worthwhile to notice that thermal effects are not important for the calculation of $P_{e}$, but only for $P_{\mu}$, especially for small values of $n\left(n<0.4 \mathrm{fm}^{-3}\right)$. We found that thermal effects produce a slightly stiffer equation of state with respect to the case of cold nuclear matter. The above EOS can be applied to the evaluation of the bulk properties of hot neutron stars (mass and radius).

The study of hot nuclear matter in the absence of neutrino trapping is the first step to study the properties of hot neutron stars and supernova matter. Next, one can study the more realistic case of neutrino-trapped matter in $\beta$-equilibrium. In this case, the $\beta$-equilibrium conditions in matter are altered from the case in which neutrinos have left the system and thus the composition of matter is affected. The proton fraction increases dramatically and influences significantly the properties of nuclear matter. Such a work is in progress. 


\section{Summary}

The evaluation of the equation of state of hot nuclear matter is an important problem in nuclear physics and astrophysics. EOS is the basis ingredient for the study of the supernova explosion as well as on determining the properties of hot neutron stars. The motivation of the present work is to apply a momentum-dependent interaction model for the study of the hot nuclear matter EOS under $\beta$-equilibrium in order to be able to study simultaneously thermal effects, not only on the kinetic part of the symmetry energy and symmetry free energy, but also on the interaction part of the above quantities as well. We calculate the proton fraction, as well as the lepton fractions, by applying the constraints for chemical equilibrium and charge neutrality. The free energy, the internal energy and also the pressure are calculated as functions of baryon density and for various values of temperature. We also concentrate on the evaluation of thermal effects on the internal energy and baryon pressure. Special attention is dedicated to the study of the contribution of the components of $\beta$-stable nuclear matter on the entropy per particle, a quantity of great interest in the study of structure and collapse of supernova. We present and analyze the contribution of each component. Finally, we present the EOS of $\beta$-stable hot nuclear matter, by taking into account and analyzing the contributions to the total pressure of each component. The above EOS can be applied to the evaluation of the gross properties of hot neutron stars i.e. mass and radius, (work in progress).

\section{Acknowledgments}

One of the authors (Ch.C.M) would like to thank Professor Tatsuyauki Takatsuka for valuable comments and correspondence.

\section{Appendix}

The energy density of baryons (Eq. (1) ), at $T=0$, is given by

$$
\begin{aligned}
\epsilon(n, I, T=0) & =\frac{3}{10} E_{F}^{0} n_{0} u^{5 / 3}\left[(1+I)^{5 / 3}+(1-I)^{5 / 3}\right]+\frac{1}{3} A n_{0}\left[\frac{3}{2}-\left(\frac{1}{2}+x_{0}\right) I^{2}\right] u^{2} \\
& +\frac{\frac{2}{3} B n_{0}\left[\frac{3}{2}-\left(\frac{1}{2}+x_{3}\right) I^{2}\right] u^{\sigma+1}}{1+\frac{2}{3} B^{\prime}\left[\frac{3}{2}-\left(\frac{1}{2}+x_{3}\right) I^{2}\right] u^{\sigma-1}} \\
& +\frac{3}{2} n_{0} u \sum_{i=1,2}\left[C_{i}+\frac{C_{i}-8 Z_{i}}{5} I\right]\left(\frac{\Lambda_{i}}{k_{F}^{0}}\right)^{3}\left(\frac{((1+I) u)^{1 / 3}}{\frac{\Lambda_{i}}{k_{F}^{0}}}-\tan ^{-1} \frac{((1+I) u)^{1 / 3}}{\frac{\Lambda_{i}}{k_{F}^{0}}}\right) \\
& +\frac{3}{2} n_{0} u \sum_{i=1,2}\left[C_{i}-\frac{C_{i}-8 Z_{i}}{5} I\right]\left(\frac{\Lambda_{i}}{k_{F}^{0}}\right)^{3}\left(\frac{((1-I) u)^{1 / 3}}{\frac{\Lambda_{i}}{k_{F}^{0}}}-\tan ^{-1} \frac{((1-I) u)^{1 / 3}}{\frac{\Lambda_{i}}{k_{F}^{0}}}\right) .
\end{aligned}
$$

The pressure of the baryons, at $T=0$, defined as

$$
P=n^{2} \frac{d(\epsilon / n)}{d n}
$$

is given by

$$
P(n, I, T=0)=\frac{1}{5} n_{0} E_{F}^{0} u^{5 / 3}\left[(1+I)^{5 / 3}+(1-I)^{5 / 3}\right]+\frac{1}{3} n_{0} u^{2} A\left[\frac{3}{2}-\left(\frac{1}{2}+x_{0}\right) I^{2}\right]
$$




$$
\begin{aligned}
& +\frac{2}{3} B \sigma n_{0} u^{\sigma+1} \frac{\left[\frac{3}{2}-\left(\frac{1}{2}+x_{3}\right) I^{2}\right]\left(1+\frac{2}{3 \sigma} B^{\prime} u^{\sigma-1}\left[\frac{3}{2}-\left(\frac{1}{2}+x_{3}\right) I^{2}\right]\right)}{\left(1+\frac{2}{3} B^{\prime}\left[\frac{3}{2}-\left(\frac{1}{2}+x_{3}\right) I^{2}\right] u^{\sigma-1}\right)^{2}} \\
& +\frac{n_{0} u^{2}}{2} \sum_{i=1,2}\left[C_{i}+\frac{C_{i}-8 Z_{i}}{5} I\right]\left(\frac{\Lambda_{i}}{k_{F}^{0}}\right)^{2} \frac{(1+I)^{1 / 3}}{u^{2 / 3}}\left(1-\frac{1}{1+\frac{(1+I)^{2 / 3} u^{2 / 3}}{\left(\frac{\Lambda_{i}}{k_{F}^{0}}\right)^{2}}}\right) \\
& +\frac{n_{0} u^{2}}{2} \sum_{i=1,2}\left[C_{i}-\frac{C_{i}-8 Z_{i}}{5} I\right]\left(\frac{\Lambda_{i}}{k_{F}^{0}}\right)^{2} \frac{(1-I)^{1 / 3}}{u^{2 / 3}}\left(1-\frac{1}{1+\frac{(1-I)^{2 / 3} u^{2 / 3}}{\left(\frac{\Lambda_{i}}{k_{F}^{0}}\right)^{2}}}\right) .
\end{aligned}
$$

In Eq. (50) $E_{F}^{0}$ is the Fermi energy of symmetric nuclear matter corresponding to equilibrium density $n_{0}$ and is given by

$$
E_{F}^{0}=\frac{\left(\hbar k_{F}^{0}\right)^{2}}{2 m}, \quad k_{F}^{0}=\left(3 \pi^{2} \frac{n_{0}}{2}\right)^{1 / 3} .
$$

The Fermi momenta of protons and neutrons are

$$
\begin{gathered}
k_{F}^{p}=\left(3 \pi^{2} x n\right)^{1 / 3}=\left(3 \pi^{2} \frac{1-I}{2} n\right)^{1 / 3}, \\
k_{F}^{n}=\left(3 \pi^{2}(1-x) n\right)^{1 / 3}=\left(3 \pi^{2} \frac{1+I}{2} n\right)^{1 / 3} .
\end{gathered}
$$

The chemical potentials of protons and neutrons, at $T=0$, are given by

$$
\begin{aligned}
\mu_{n} & =E+u\left(\frac{\partial E}{\partial u}\right)_{Y_{p}}-Y_{p}\left(\frac{\partial E}{\partial Y_{p}}\right)_{n}, \\
\mu_{p} & =\mu_{n}+\left(\frac{\partial E}{\partial Y_{p}}\right)_{n}, \\
\hat{\mu} & =\mu_{n}-\mu_{p}=-\left(\frac{\partial E}{\partial Y_{p}}\right)_{n},
\end{aligned}
$$

where

$$
\begin{aligned}
\left(\frac{\partial E}{\partial u}\right)_{Y_{p}} & =\frac{1}{5} E_{F}^{0} u^{-1 / 3}\left[(1+I)^{5 / 3}+(1-I)^{5 / 3}\right]+\frac{1}{3} A\left[\frac{3}{2}-\left(\frac{1}{2}+x_{0}\right) I^{2}\right] \\
& +\frac{\frac{2}{3} B u^{\sigma-1} \sigma\left[\frac{3}{2}-\left(\frac{1}{2}+x_{3}\right) I^{2}\right]\left(1+\frac{2}{3 \sigma} B^{\prime} u^{\sigma-1}\left[\frac{3}{2}-\left(\frac{1}{2}+x_{3}\right) I^{2}\right]\right)}{\left(1+\frac{2}{3} B^{\prime} u^{\sigma-1}\left[\frac{3}{2}-\left(\frac{1}{2}+x_{3}\right) I^{2}\right]\right)^{2}} \\
& +\frac{1}{2} \sum_{i=1,2}\left[C_{i}+\frac{C_{i}-8 Z_{i}}{5} I\right]\left(\frac{\Lambda_{i}}{k_{F}^{0}}\right)^{2} \frac{(1+I)^{1 / 3}}{u^{2 / 3}}\left(1-\frac{1}{1+\frac{(1+I)^{2 / 3} u^{2 / 3}}{\left(\frac{\Lambda_{i}}{k_{F}^{\circ}}\right)^{2}}}\right)
\end{aligned}
$$




$$
\begin{aligned}
& +\frac{1}{2} \sum_{i=1,2}\left[C_{i}-\frac{C_{i}-8 Z_{i}}{5} I\right]\left(\frac{\Lambda_{i}}{k_{F}^{0}}\right)^{2} \frac{(1-I)^{1 / 3}}{u^{2 / 3}}\left(1-\frac{1}{1+\frac{(1-I)^{2 / 3} u^{2 / 3}}{\left(\frac{\Lambda_{i}}{k_{F}^{0}}\right)^{2}}}\right) \\
& \left(\frac{\partial E}{\partial Y_{p}}\right)_{n}=\frac{1}{2} E_{F}^{0} u^{2 / 3}\left[(1+I)^{2 / 3}-(1-I)^{2 / 3}\right]-\frac{1}{3} A u\left(\frac{1}{2}+x_{0}\right) I \\
& -\frac{\frac{2}{3} B u^{\sigma}\left(\frac{1}{2}+x_{3}\right) I}{\left(1+\frac{2}{3} B^{\prime} u^{\sigma-1}\left[\frac{3}{2}-\left(\frac{1}{2}+x_{3}\right) I^{2}\right]\right)^{2}} \\
& +\frac{3}{2} \sum_{i=1,2}\left[\frac{C_{i}-8 Z_{i}}{5} I\right]\left(\frac{\Lambda_{i}}{k_{F}^{0}}\right)^{3}\left(\frac{((1+I) u)^{1 / 3}}{\frac{\Lambda_{i}}{k_{F}^{0}}}-\tan ^{-1} \frac{((1+I) u)^{1 / 3}}{\frac{\Lambda_{i}}{k_{F}^{0}}}\right) \\
& -\frac{3}{2} \sum_{i=1,2}\left[\frac{C_{i}-8 Z_{i}}{5} I\right]\left(\frac{\Lambda_{i}}{k_{F}^{0}}\right)^{3}\left(\frac{((1-I) u)^{1 / 3}}{\frac{\Lambda_{i}}{k_{F}^{0}}}-\tan ^{-1} \frac{((1-I) u)^{1 / 3}}{\frac{\Lambda_{i}}{k_{F}^{0}}}\right) \\
& +\frac{1}{2} \sum_{i=1,2}\left[C_{i}+\frac{C_{i}-8 Z_{i}}{5} I\right]\left(\frac{\Lambda_{i}}{k_{F}^{0}}\right)^{2} \frac{u^{1 / 3}}{(1+I)^{2 / 3}}\left(1-\frac{1}{1+\frac{(1+I)^{2 / 3} u^{2 / 3}}{\left(\frac{\Lambda_{i}}{k_{F}^{0}}\right)^{2}}}\right) \\
& -\frac{1}{2} \sum_{i=1,2}\left[C_{i}-\frac{C_{i}-8 Z_{i}}{5} I\right]\left(\frac{\Lambda_{i}}{k_{F}^{0}}\right)^{2} \frac{u^{1 / 3}}{(1-I)^{2 / 3}}\left(1-\frac{1}{1+\frac{(1-I)^{2 / 3} u^{2 / 3}}{\left(\frac{\Lambda_{i}}{k_{F}^{0}}\right)^{2}}}\right) .
\end{aligned}
$$

\section{References}

[1] H.A. Bethe, Rev. Mod. Phys. 62, 801 (1990); H.A. Bethe, Ann. Rev. Nucl. Part. Sci. 38, 1 (1988).

[2] Madappa Prakash, I. Bombaci, Manju Prakash, P.J. Ellis, J.M. Lattimer, R. Knorren, Phys. Rep. 280, 1 (1997).

[3] B.A. Li, and W. Udo Schröder, Isospin Physics in Heavy-Ion Collisions at Intermediate Energies (New York: Nova Science) (2001).

[4] B.A. Li, L.W. Chen, and C.M. Ko, Phys. Rep. 464, 113 (2008).

[5] G. Baym, H.A. Bethe, and C.J. Pethick, Nucl. Phys. A 175, 225 (1971).

[6] H.A. Bethe, G.E. Brown, J. Applegate, and J.M. Lattimer, Nucl. Phys. A 324, 487 (1979).

[7] B. Friedman and V.R. Pandharipande, Nucl. Phys. A 361, 502 (1981).

[8] J.M. Lattimer and F.D. Swesty, Nucl. Phys. A 535, 331 (1991).

[9] T. Takatsuka, S. Nishizaki, and J. Hiura, Progr. of Theor. Phys. 92, 779 (1994). 
[10] T. Takatsuka, Progr. of Theor. Phys. 95, 901 (1996).

[11] C. Das, R. Sahu, and A. Mishra, Phys. Rev. C 75, 015807 (2007).

[12] Bernard ter Haar and R. Malfliet, Phys. Rev. Lett. 56, 1237 (1986).

[13] I. Bombaci, Madappa Prakash, Manju Prakash, P.J Ellis, J.M. Lattimer, and G.E. Brown, Nucl. Phys. A 583, 623 (1995).

[14] W.A. Küpper, G. Wegmann, and E.R. Hilf, Ann. of Phys. 88, 454 (1974).

[15] J.M. Lattimer and D.G. Ravenhall, Astr. Jour. 223, 314 (1978).

[16] M.F. El Eid and W. Hillebrandt, Astron. Astrophy. Suppl. Ser. 42, 215 (1980).

[17] P. Lamb, Mon. Not. R. Astr. Soc. 188, 565 (1979).

[18] H.M. Antia, B. Banerjee, and S.M. Chitre, Astr. Sp. Scien. 69, 471 (1980).

[19] M. Onsi, H. Przysiezniak, and J.M. Pearson, Phys. Rev. C 50, 460 (1994).

[20] K. Sumiyoshi and H. Toki, Astr. Jour. 422, 700 (1994).

[21] J.M. Lattimer, C.J. Pethick, D.G. Ravenhall, and D.Q. Lamb, Nucl. Phys. A 432, 646 (1985).

[22] H. Kanzawa, K. Oyamatsu, K. Sumiyoshi, and M. Takano, Nucl. Phys. A 791, 232 (2007).

[23] M. Modarres, J. Phys. G: Nucl. Part. Phys. 23, 923 (1997); M. Modarres, J. Phys. G: Nucl. Part. Phys. 21, 351 (1995); M. Modarres, J. Phys. G: Nucl. Part. Phys. 19, 1349 (1993).

[24] R. Manka, I. Bednarek, and G. Przybyla, Phys. Rev. C 62, 015802 (2000).

[25] W. Zuo, Z.H. Li, A. Li, G.C. Lu, Phys. Rev. C 69, 064001 (2003).

[26] L.W. Chen, F.S. Zhang, Z.H. Lu, W.F. Li, Z.Y. Zhu, H.R. Ma, J. Phys. G: Nucl. Part. Phys. 27, 1799 (2001).

[27] V.K. Mishra, G. Fai, L.P. Csernai, E. Osnes, Phys. Rev. C 47, 1519 (1993).

[28] L.P. Csernai, G. Fai, C. Gale, E. Osnes, Phys. Rev. C 46, 736 (1992).

[29] S.J. Lee, A.Z. Mekjian, Phys. Rev. C 63, 044605 (2001); A.Z. Mekjian, S.J. Lee, L. Zamick, Phys. Rev. C 72, 044305 (2005); A.Z. Mekjian, S.J. Lee, L. Zamick, Phys. Lett. B 621, 239 (2005); S.J. Lee, A.Z. Mekjian, Phys. Rev. C 77, 054612 (2008).

[30] H. Müller and B.D. Serot, Phys. Rev. C 52, 2072 (1995).

[31] M. Baldo and Ferreira, Phys. Rev. C 59, 682 (1999).

[32] G.F. Burgio, M. Baldo, O.E. Nicotra, and H.J. Schulze, Astrophys. Space Sci. 308, 387 (2007); O.E. Nicotra, M. Baldo, G.F. Burgio, and H.J. Schulze, Astron. and Astroph. 451, $213(2006)$.

[33] J. Xu, L.W. Chen, B.A. Li, H.R. Ma, Phys. Rev. C 75, 014607 (2007).

[34] P. Wang, Phys. Rev. C 61, 054904 (2000). 
[35] P.K. Jena, and L.P. Singh, Phys. Rev. C 70, 045803 (2004).

[36] J.N. De, N. Rudra, S. Pal, and S.K. Samaddar, Phys. Rev. C 53, 780 (1996); T. Sil, B.K. Agrawal, J.N. De, and S.K. Samaddar, Phys. Rev. C 63, 054604 (2001); S.K. Samaddar, J.N. De, X. Viñas, and M. Centelles, Phys. Rev. C 76, 041602 (R) (2007).

[37] L. Tolos, B. Frieman, and A. Schwenk, Nucl. Phys. A 806, 105 (2008).

[38] M. Abd-Alla, H.S. Ragab, and M.Y.M. Hassan, Acta Phys. Pol. B 24, 1519 (1993).

[39] Ch.C. Moustakidis, Phys. Rev. C 76, 025805 (2007).

[40] Ch.C. Moustakidis, Phys. Rev. C 78, 054323 (2008).

[41] R.B. Wiringa, V. Fiks, and A. Fabrocini, Phys. Rev. C 38, 1010 (1988).

[42] A. Akmal, V.R. Pandharipande, and D.G. Ravenhall, Phys. Rev. C 58, 1804 (1998).

[43] G.F. Bertsch and S. Das Gupta, Phys. Rep. 160, 189 (1988).

[44] C. Gale, G.F. Bertsch, and S. Das Gupta, Phys. Rev. C 35, 1666 (1987).

[45] M. Prakash, T.T.S. Kuo, and S. Das Gupta, Phys. Rev. C 37, 2253 (1988); G.M. Welke, M. Prakash, T.T.S. Kuo, S. Das Gupta, and C. Gale, Phys. Rev. C 38, 2101 (1988);C. Gale, G.M. Welke, M. Prakash, S.J. Lee, and S. Das Gupta, Phys. Rev. C 41, 1545 (1990);

[46] B.A. Li, C.B. Das, S. Das Gupta, and C. Gale, Phys. Rev. C 69, 011603(R) (2004); C.B. Das, S. Das Gupta, C. Gale, and B.A. Li, Phys. Rev. C 67, 034611 (2003); L.W. Chen, C.M. Ko, and B.A. Li, Phys. Rev. C 69, 054606 (2004); B.A. Li, C.B. Das, S. Das Gupta, and C. Gale, Nucl. Phys. A 735, 563 (2004); B.A. Li, L.W. Chen, G.C. Yong, and W. Zuo, Phys. Lett. B 634, 378 (2006); B.A. Li and A.W. Steiner, Phys. Lett. B 642, 436 (2006).

[47] R.B. Wiringa, Phys. Rev. C 38, 2707 (1988).

[48] G.Q. Li and R. Machleidt, Phys. Rev. C 48, 2967 (1993).

[49] M. Kutschera, Phys. Lett. B 340, 1 (1994); M. Kutschera, Z. Phys. A 348, 263 (1994); M. Kutschera, Acta Phys. Pol. B 29, 25 (1998); S. Kubis, M. Kutschera, Nucl. Phys. A 720, 189 (2003).

[50] B.A. Li, Phys. Rev. Lett. 88, 192701 (2002).

[51] C. Fuchs and H.H. Wolter, Eur. Phys. J. A 30, 5 (2006).

[52] N.K. Glendenning, Compact Stars: Nuclear Physics, Particle Physics, and General Relativity, Springer-Verlag, New York, 1997.

[53] R.B. Wiringa, V. Fiks, and A. Fabrocini, Phys. Rev. C 38, 1010 (1988).

[54] D.V. Shetty, S.J. Yennello, G.A Souliotis, Phys. Rev. C 75, 34602 (2007); D.V. Shetty, S.J. Yennello, G.A Souliotis, Phys. Rev. C 76, 024606 (2007).

[55] L.W. Chen, C.M. Ko, B.A. Li, Phys. Rev. Lett. 94, 032701 (2005).

[56] M.B. Tsang et al., Phys. Rev. Lett. 92, 062701 (2004). 
[57] C.H. Lee, T.T.S. Kuo, G.Q. Li, and G.E. Brown, Phys. Rev. C 57, 3488 (1998).

[58] F. Sammarruca and P. Liu, arXiV: 0806.1936 [nucl-th](2008).

[59] V.P. Psonis, Ch.C. Moustakidis, and S.E. Massen, Mod. Phys. Lett. A 22, 1233 (2007).

[60] M. Prakash, The Equation of State and Neutron Stars lectures delivered at the Winter School held in Puri India (1994).

[61] B.A. Li, C.M. Kuo, Z.Z. Ren, Phys. Rev. Lett. 78, 1644 (1997).

[62] V. Baran, M. Colonna, V. Greco, and M. Di Toro, Phys. Rep. 410, 335 (2005).

[63] D.L. Goodstein, States of Matter (Dover, New York, 1985).

[64] A.L. Fetter and J.D. Walecka, Quantum Theory of Many-Particle Systems (Dover Publications, Mineola, New York, 2003). 

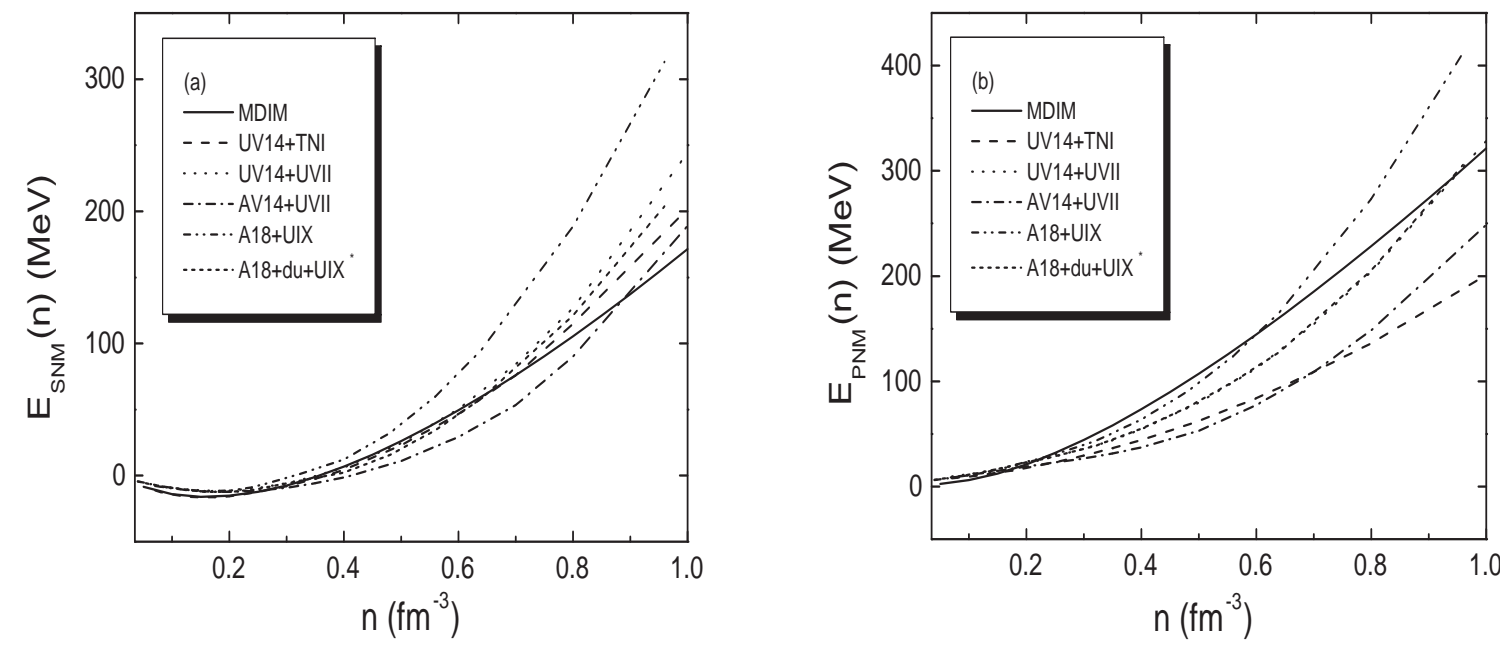

Figure 1: Energy per baryon of symmetric (a) and pure neutron matter (b) of the present model (MDIM) in comparison with those originated from realistic calculations. More details for the models UV14+TNI, UV14+UVII and AV14+UVII in Ref. [41] and for the models A18+UIX and $\mathrm{A} 18+\mathrm{du}+\mathrm{UIX}^{*}$ in Ref. [42].
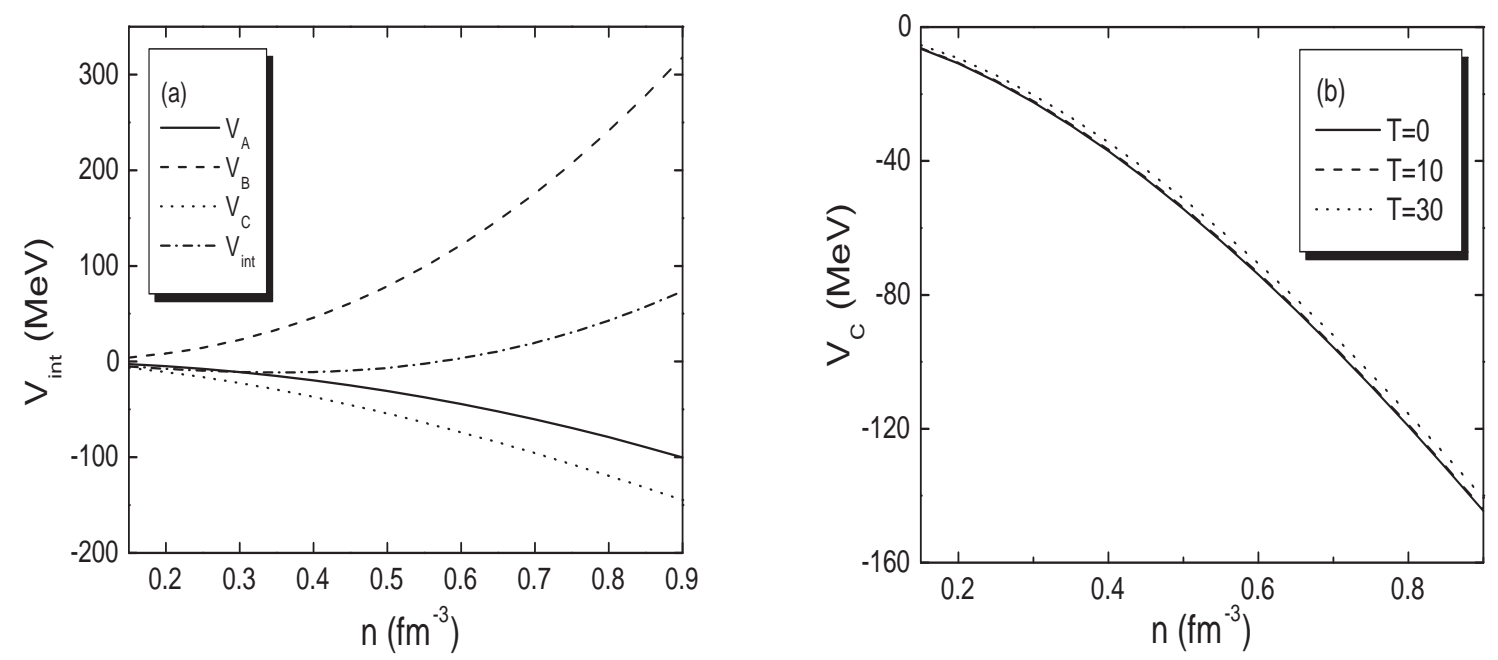

Figure 2: a) The contribution of the various terms $V_{A}, V_{B}, V_{C}$ and the total potential energy density $V_{\text {int }}$ as a function of the baryon density b) The momentum dependent term $V_{C}$ as a function of the baryon density at temperature $T=0, T=10$ and $T=30 \mathrm{MeV}$. 

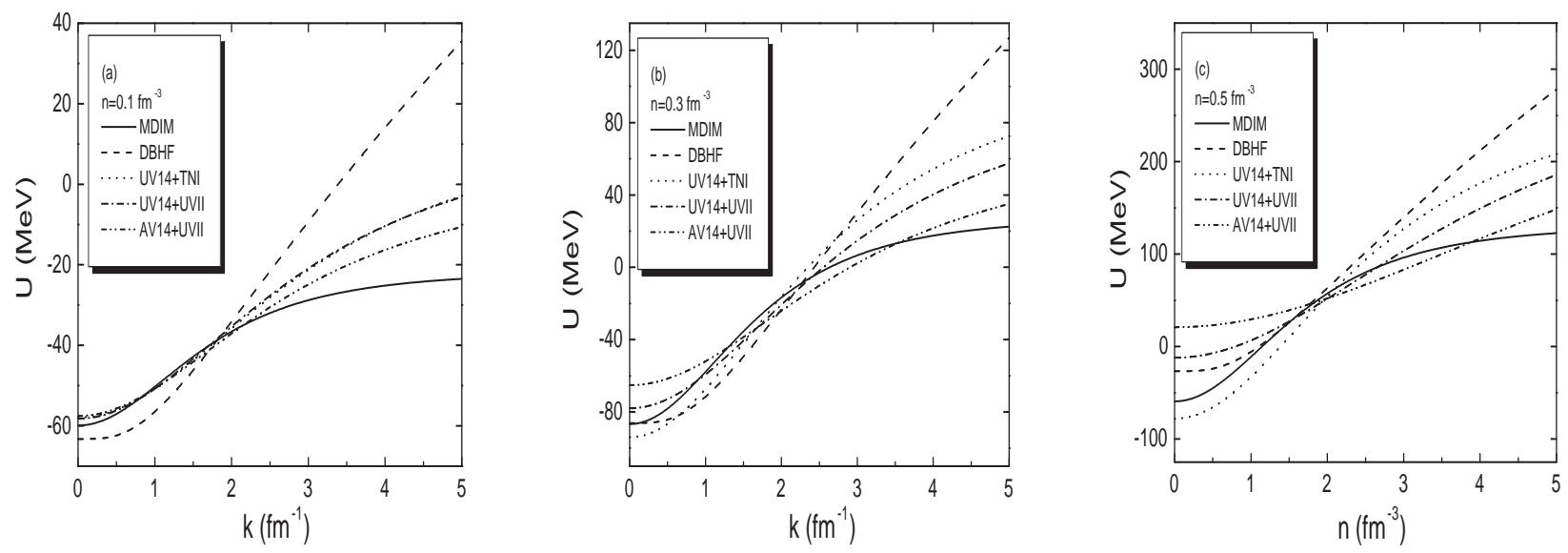

Figure 3: A comparison of the single-particle potential of symmetric nuclear matter from the present model (MDIM) with the microscopic calculations of Wirigna [47] and Li et al. [48], for densities $n=0.1 \mathrm{fm}^{-3}, n=0.3 \mathrm{fm}^{-3}$ and $n=0.5 \mathrm{fm}^{-3}$.
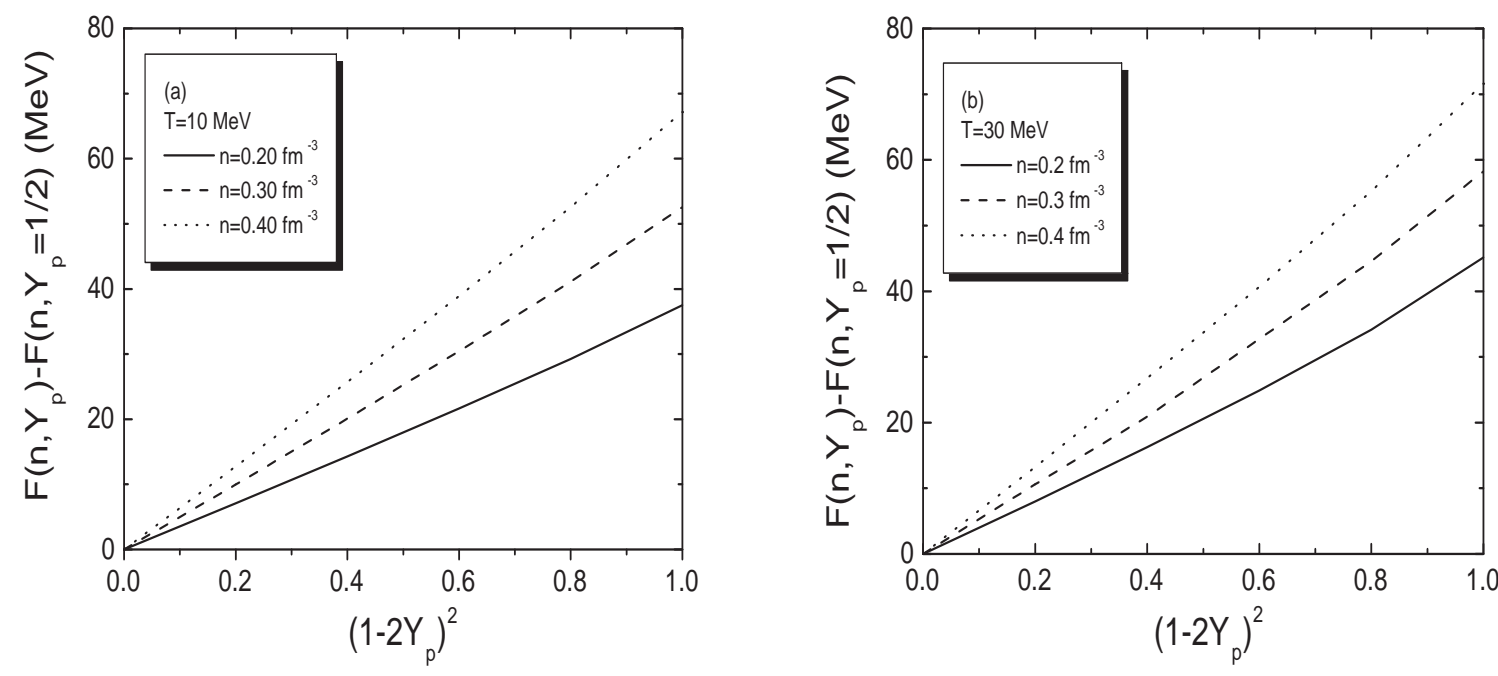

Figure 4: The difference $F\left(n, T, Y_{p}\right)-F\left(n, T, Y_{p}=1 / 2\right)$ as a function of $\left(1-2 Y_{p}\right)^{2}$ at temperatures a) $T=10$ and b) $T=30 \mathrm{MeV}$, for three baryon densities. 


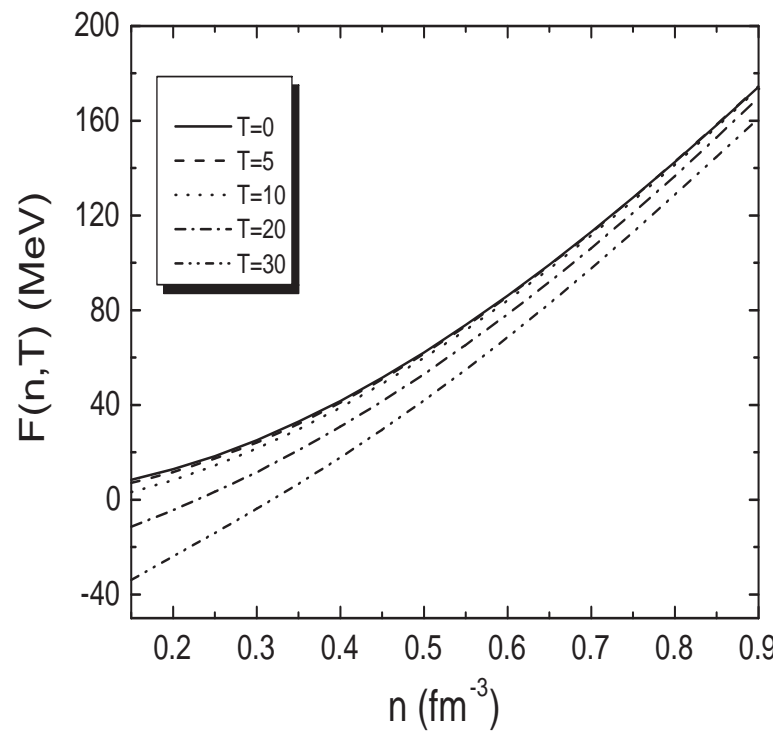

Figure 5: The Helmholtz free energy $F(n, T, I)$ of $\beta$-stable matter versus the baryon density $n$, for various values of $T$ (in $\mathrm{MeV}$ ).
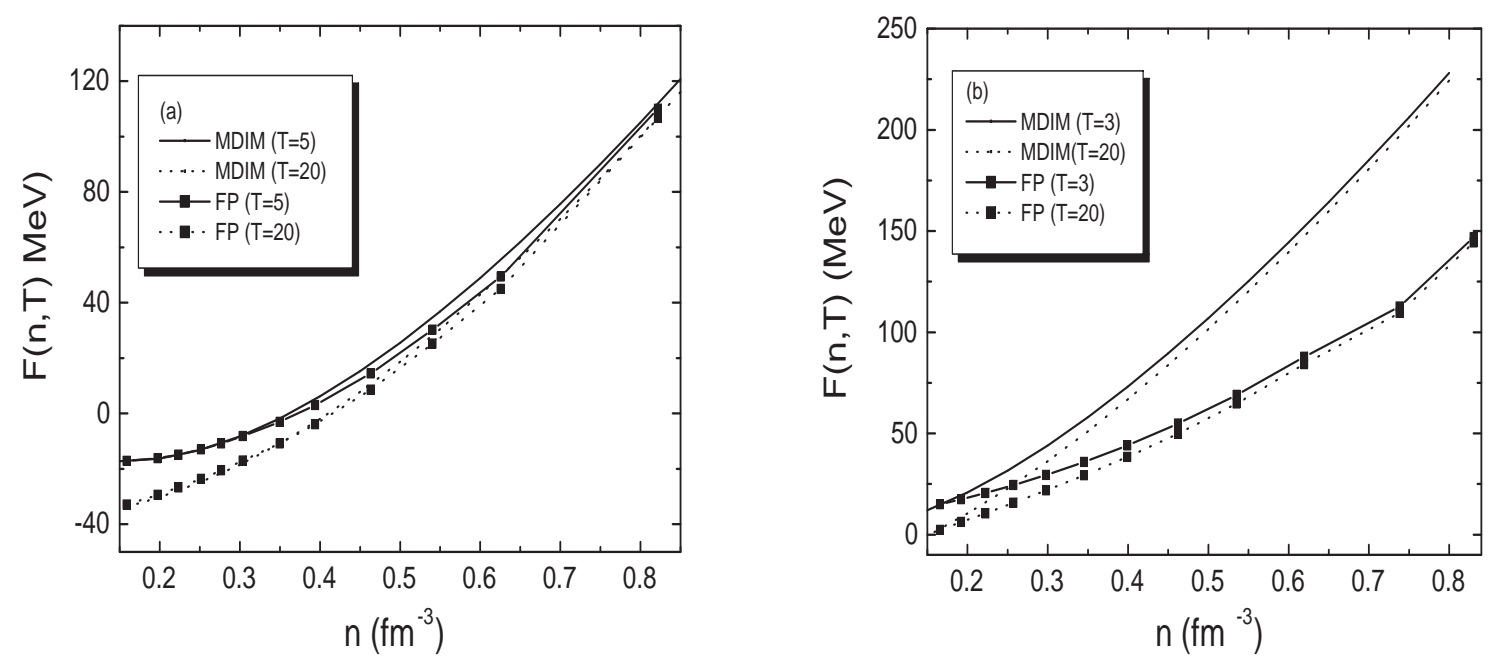

Figure 6: (a) The free energy of symmetric nuclear matter (for $T=5 \mathrm{MeV}$ and $T=20 \mathrm{MeV}$ ) and (b) for pure neutron matter (for $T=3 \mathrm{MeV}$ and $T=20 \mathrm{MeV}$ ) of the proposed model (MDIM) in comparison with the free energy calculated by Friedman and Pandharipande model (FP) [7. 


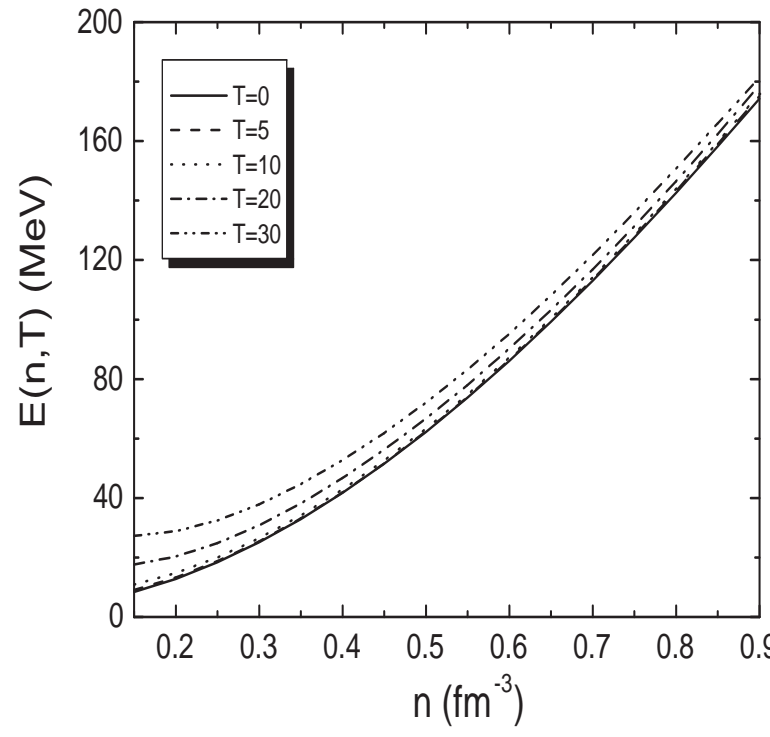

Figure 7: The internal energy $E(n, T)$ of $\beta$-stable matter as a function of the baryon density $n$ for various values of $T$.

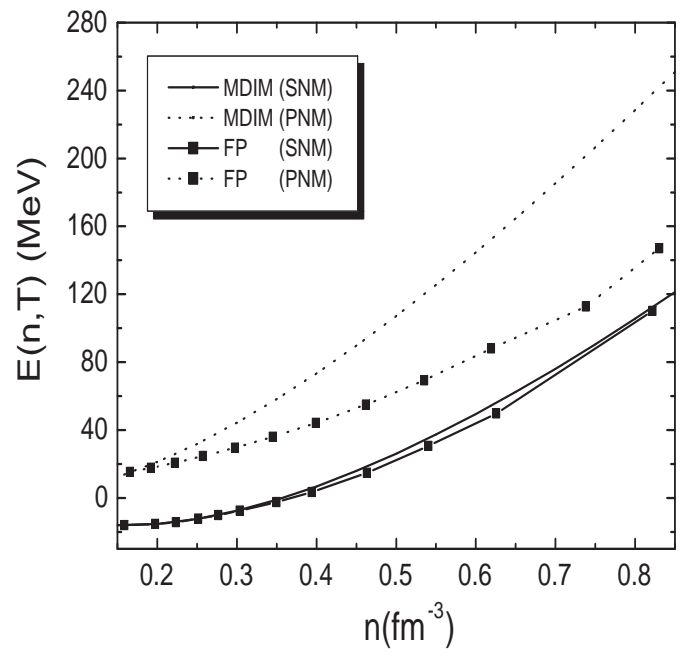

Figure 8: The internal energy (for $T=0 \mathrm{MeV}$ ) for symmetric nuclear matter (SNM) and pure neutron matter (PNM) calculated with the MDIM in comparison with the FP model. 


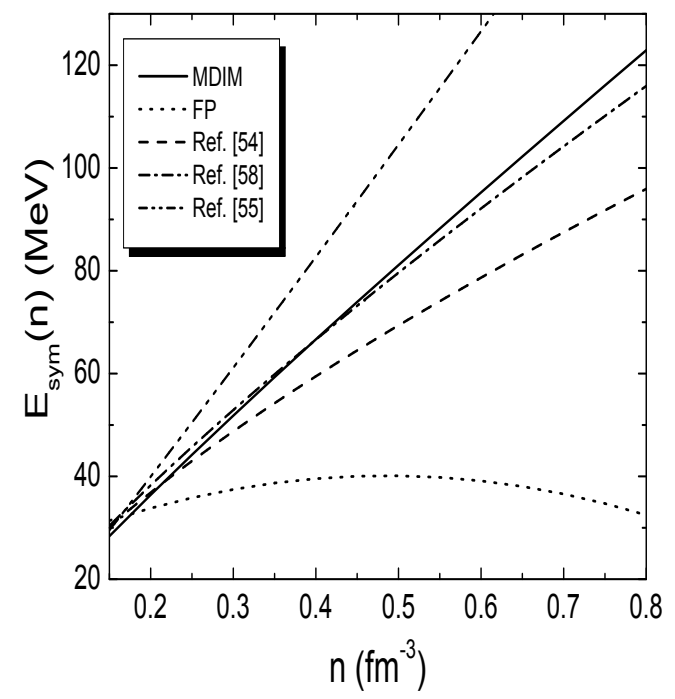

Figure 9: The nuclear symmetry energy calculated with the MDIM in comparison with the FP model as well as the results of Refs. [54], [58] and [55].

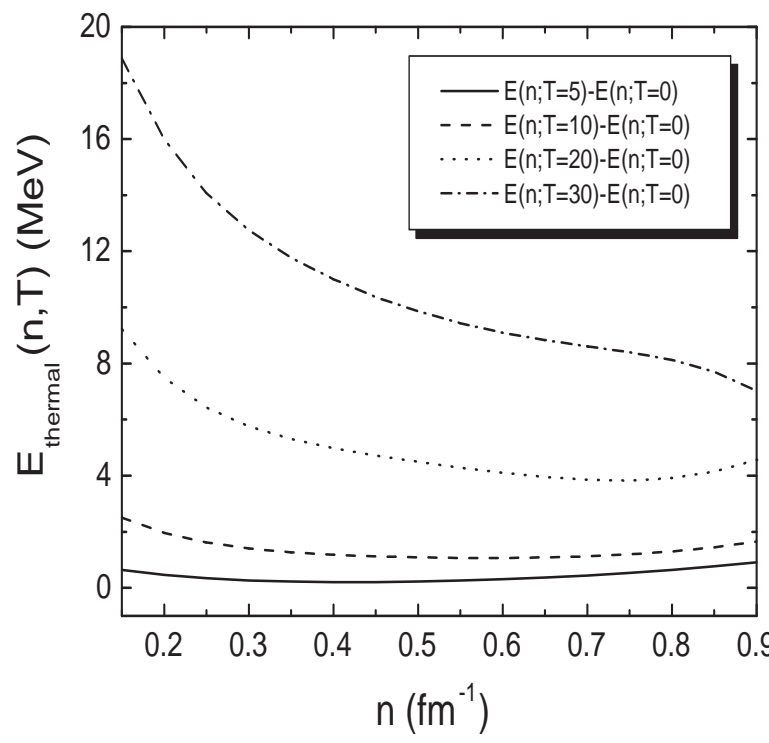

Figure 10: The thermal energy $E_{\text {thermal }}(n, T)=E(n, T)-E(n, T=0)$ of $\beta$-stable matter versus the baryon density $n$, for various values of $T$. 

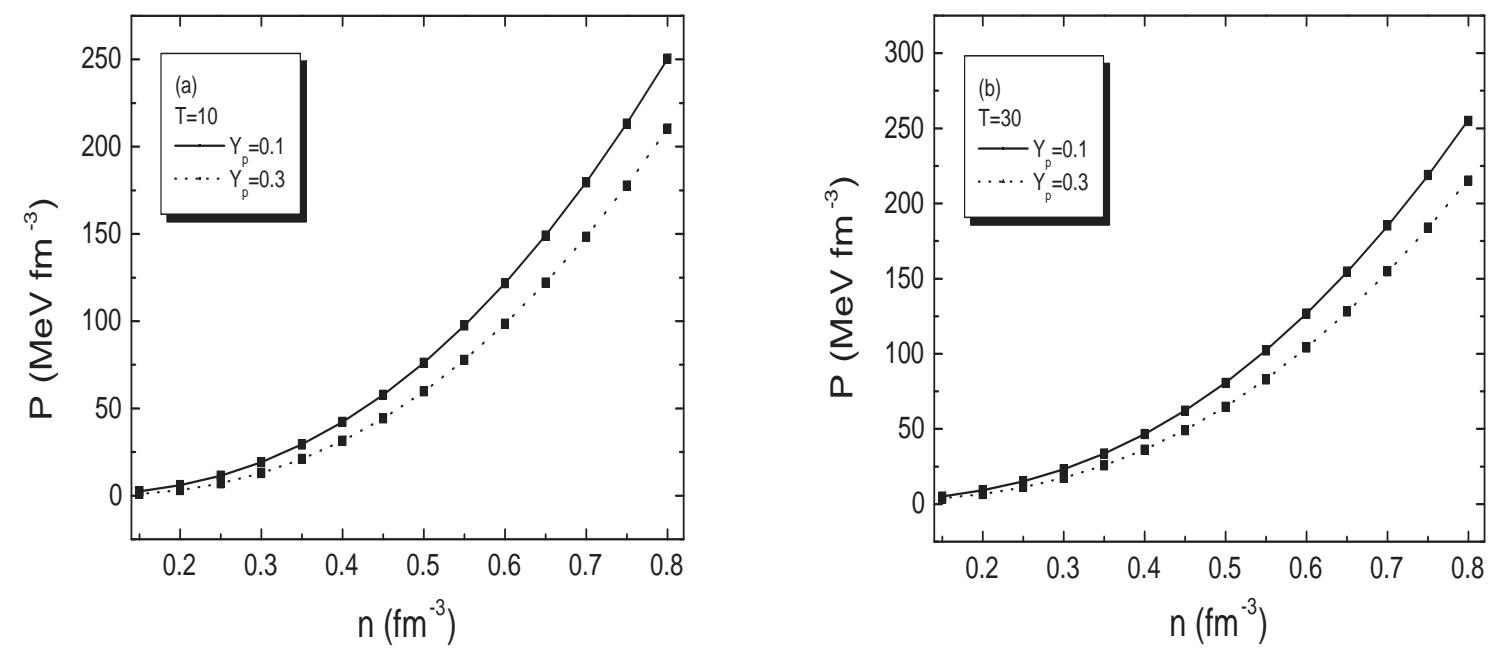

Figure 11: The pressure $P$ of asymmetric nuclear matter for $Y_{p}=0.1$ and 0.3 at a) $T=10$ and b) $30 \mathrm{MeV}$. The full lines give the results calculated from Eq. (24), while the squares represent results obtained by differentiating $F(n, T)($ Eq. (23) $)$.

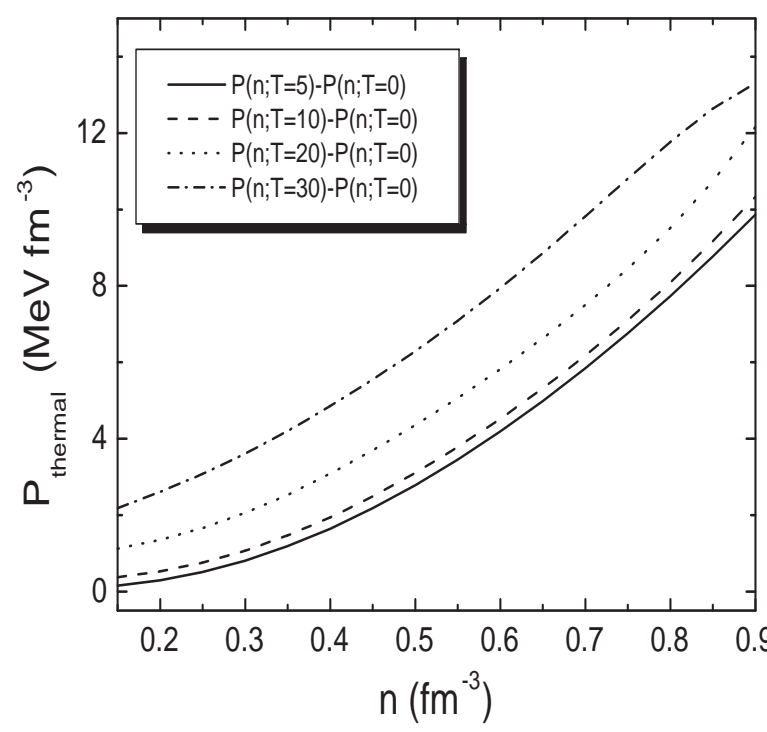

Figure 12: The thermal pressure $P_{\text {thermal }}(n, T)=P(n, T)-P(n, T=0)$ of $\beta$-stable matter versus the baryon density $n$, for various values of $T$. 


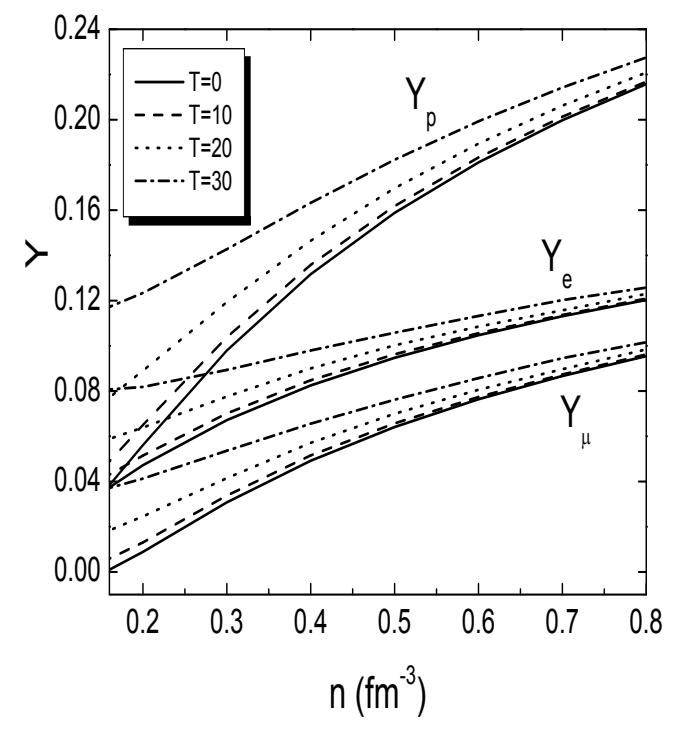

Figure 13: The fractions of protons $Y_{p}$ electrons $Y_{e}$ and muons $Y_{\mu}$ of $\beta$-stable matter as functions of the baryon density $n$, for various values of $T$.
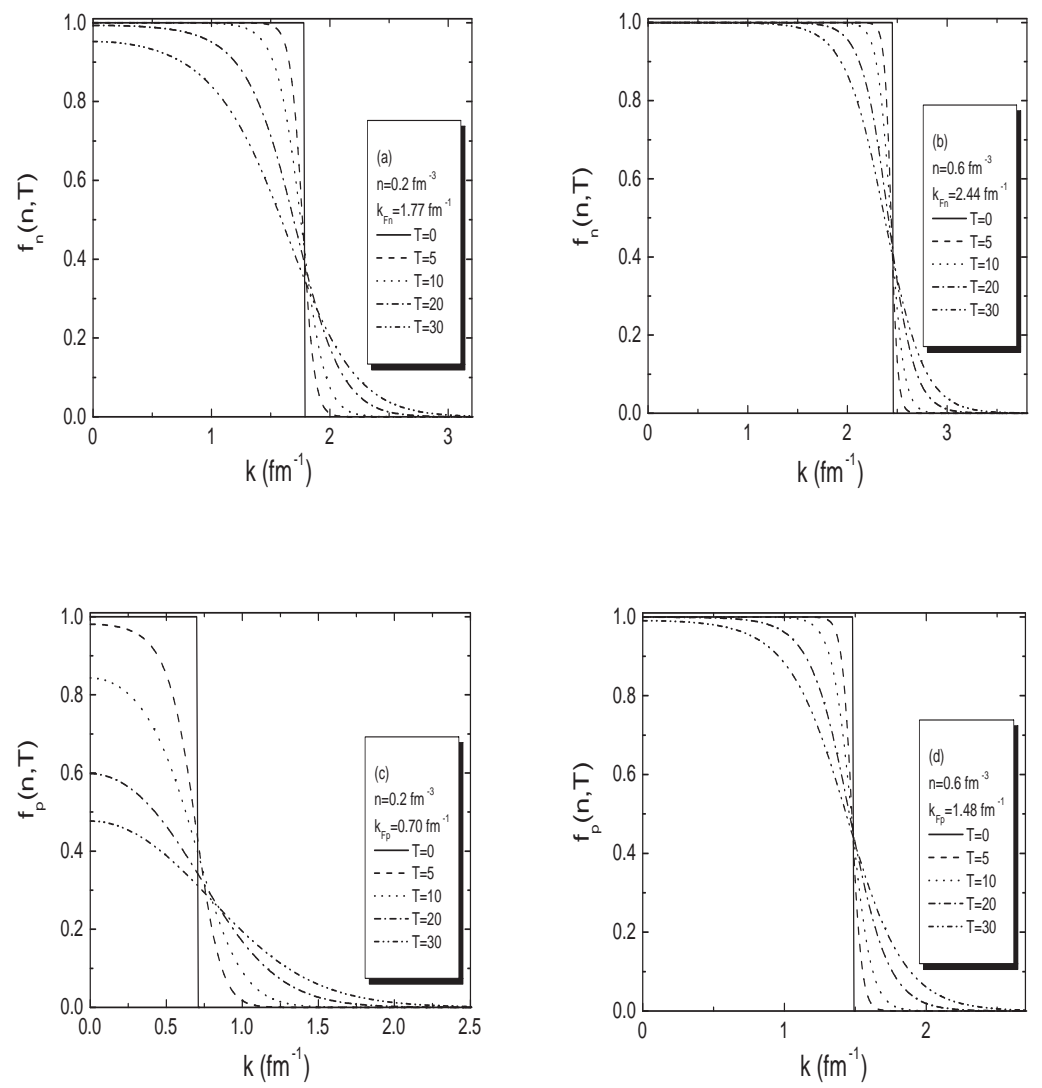

Figure 14: The Fermi-Dirac distribution function $f_{\tau}(n, T)$ for protons and neutrons $(\tau=p, n$ respectively), for $n=0.2 \mathrm{fm}^{-3}, n=0.4 \mathrm{fm}^{-3}$ and $n=0.6 \mathrm{fm}^{-3}$ and various values of $T$. 


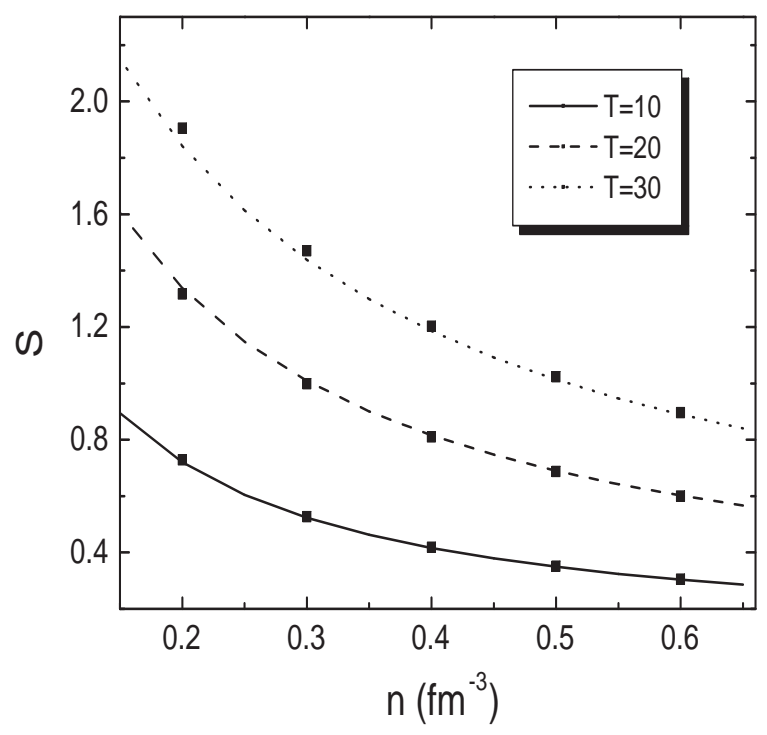

Figure 15: The entropy per particle $S$ of asymmetric nuclear matter with $Y_{p}=0.2$ at $T=10,20,30$ $\mathrm{MeV}$. The full lines give the entropy calculated from Eq. (21), while the squares give results obtained by differentiating $F(n, T)$ (Eq. (25)) .

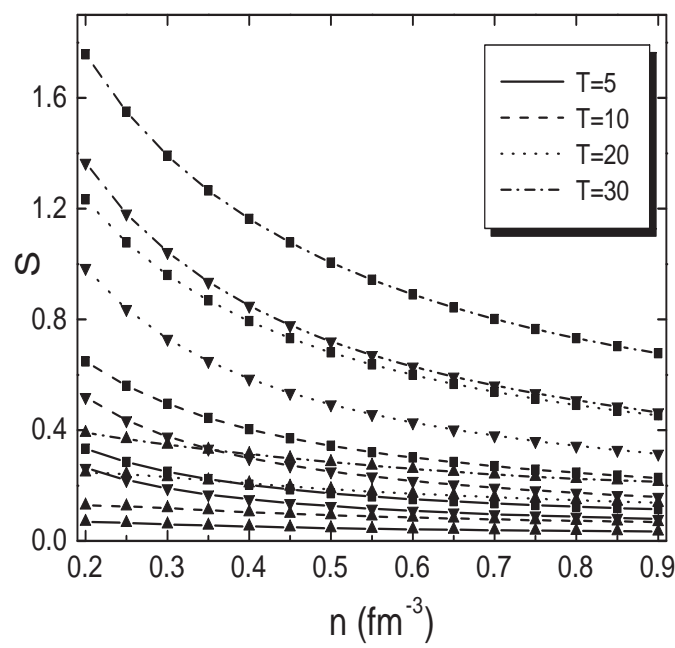

Figure 16: Contributions to the total entropy per particle of protons $\left(S_{p}\right)$ (up triangles) neutrons $\left(S_{n}\right)$ (upside down triangles) and the total entropy $\left(S_{b}\right)$ (squares). 


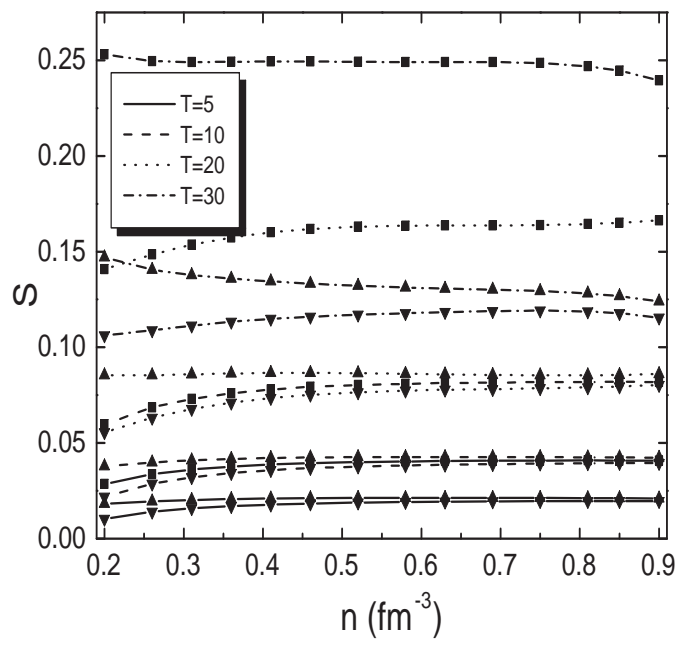

Figure 17: Contributions to the total entropy per particle of electrons $\left(S_{e}\right)$ (up triangles) muons $\left(S_{\mu}\right)$ (upside down triangles) and the total $S_{l}$ (squares).

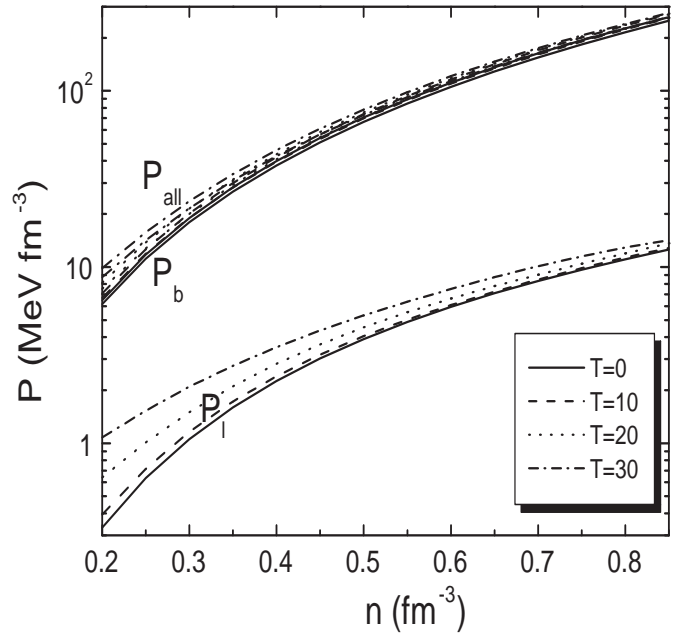

Figure 18: The pressures of baryons $P_{b}$ leptons $P_{l}$ (electrons+muons) and the total pressure $P$ versus the baryon density, $n$ for various values of $T$. 